\title{
Mobile Social Physiology as the Future of Relationship Research and Therapy: Presentation of the Bio-App for Bonding (BAB)
}

Kyra Frederiks ${ }^{1,2}$, Paula Sterkenburg ${ }^{2,3}$, Yizhar Lavner ${ }^{4}$, Rami Cohen $^{5}$, Dima Ruinskiy ${ }^{6}$, Willem Verbeke ${ }^{7}$, Hans IJzerman ${ }^{8}$

This manuscript is not yet published. Cite at own risk!

${ }^{1}$ Industrial Design Department of the Eindhoven University of Technology, The Netherlands

${ }^{2}$ Clinical Child \& Family Studies of the Vrije Universiteit Amsterdam, The

Netherlands \& Amsterdam Public Health research institute, The Netherlands

${ }^{3}$ Bartiméus, Department of Psychotherapy, Doorn, The Netherlands

${ }^{4}$ Department of Computer Science, Tel- Hai College, Israel

${ }^{5}$ The Andrew and Erna Viterbi faculty of Electrical Engineering, Technion - Israel Institute of Technology, Israel

${ }^{6}$ Intel Corporation, Haifa, Israel

${ }^{7}$ Erasmus Research Institute of Management, Erasmus University Rotterdam

${ }^{8}$ LIP/PC2S, Université Grenoble Alpes, France

Author note: Correspondence concerning this article should be addressed to Hans IJzerman, LIP/PC2S, Université Grenoble Alpes, France, h.ijzerman@gmail.com. We thank Eli Finkel, John Bargh, Loe Feijs, and Carlo Schuengel for their helpful feedback. Any remaining errors are the authors' responsibility.

Funding Note: This research was supported by a Veni grant of the Netherlands Organization for Scientific Research (NWO) (016.145.049) and a French National Research Agency "Investissements d'avenir" program grant (ANR-15-IDEX-02) both awarded to Hans IJzerman. 


\begin{abstract}
Psychological science is in a transitional period: Many findings do not replicate and theories appear not as robust as previously presumed. We suspect that a main reason for theories not appearing as robust is because they are too simple. In this paper, we provide an important step towards this transition in the field of interpersonal relationship research by 1) providing an overarching theoretical framework grounded in existing relationship science, and 2) outlining a novel approach - mobile social physiology - that relies on intelligent technologies like wearable sensors, actuators, and modern analytical methods. At the core of our theoretical principles is co-regulation (one partner's [statistical] co-dependency on the other partner). Co-regulation has long existed in the literature, but has to date been largely untested. To test the outlined principles, we 3) present a newly programmed app - the Bio-App for Bonding (available on GitHub: https://github.com/co-relab/bioapp). By providing a paradigm shift for relationship research, the field can not only increase the accuracy of measurement and the generalizability of findings, it also allows for moving from the lab to real life situations. We discuss how the mobile social physiology approach is rooted in existing theoretical principles (e.g., Social Baseline and Attachment Theory), extends the concept of co-regulation to allow for specific measurements, and provides a research agenda to develop a model of interpersonal relationships that we hope will stand the test of time.
\end{abstract}




\section{Mobile Social Physiology as the Future of Relationship Research and Therapy: Presentation of the Bio-App for Bonding (BAB)}

The study of human behavior is arguably one of the hardest sciences (Srivastava, 2016), since many of its components, like physiology, inner mental processes, social behaviors, and culture, are highly interrelated and dynamic. Yet research concerning human behavior, in the broad sense and in the specific context of interpersonal relationships, generally occurs in a relatively isolated context: the lab. As a result, these studies are often left non-generalized towards real-life and unexamined in practice. Although lab studies allow us to formalize causality in our theories, the question remains to what extent these models are accurate depictions of real-life behaviors and, if these models are not accurate, which steps we should take to improve models of human cognition and behavior. The central aim of this article is to reconsider the accuracy of human behavior models and provide a paradigm shift specifically for relationship science through advanced methods, guided by an overarching framework on broader principles underlying interpersonal relationships. The more specific goal of this article is to describe how to move basic interpersonal relationship research from the lab to the real world.

The accuracy of models of human cognition and behavior is likely to be low. Indeed, a number of occurrences in past years have inspired reflection amongst (psychological) scientists. The first event calling this into question was a publication by Ioannidis (2005), who claimed that most published research findings are false. One of the most important events calling into question the solidity and validity of psychological science was the publication on precognition by Bem (2011), suggesting that people could foresee the future, followed by a paper on data contingent analyses by Simmons, Nelson, and Simonsohn (2011). A substantial number of large-scale replications have also identified challenges for the replicability of findings in psychology: As but three samples, for Klein et al. (2014) 10 of 
13 findings replicated; for Ebersole et al. (2016) 3 of 10; and for Klein, Vianello, et al. (2018)

14 of 28. These papers demonstrated problems related to the processes of fitting data to theories a posteriori. Generally, this process of fitting data leads to a decreased ability to predict (and thus fit) human behavior and cognition in (and to) novel situations. ${ }^{1}$

These problems likely appeared as a result of studies with too few participants (i.e., low powered), p-hacking, and publication bias (Francis, 2012), but as we will argue here they are largely constituted by inadequate theoretical reasoning, insufficient attention devoted to (modern) methods, and designs that are insufficiently representative (Brunswik, 1956). The consequence of studying humans in isolation is that interpersonal processes have been interpreted as static and mostly internal to people's mental worlds through so-called “cognitive interdependence” (Agnew, Van Lange, Rusbult, \& Langston, 1998). Lab studies on interpersonal relationships point to how thoughts about our relationships can be easily accessed, activated, and thereafter acted upon (Finkel, Rusbult, Kumashiro, \& Hannon, 2002). However, the premise that human social behavior is grounded in the social environment also implies that cognition is much more distributed and much less "inside the head". Theoretically, this premise puts the earlier position - that cognition is "in the head" into question.

We have seen problems with replication; we think that progress can now be attained by incorporating modern technological innovations in real-life studies, so that the amount of measurements (i.e., internal reliability) and the number of contexts (i.e., generalizability) dramatically increase, all the while increasing external validity and retaining the possibility to draw causal inferences. Movements away from the lab and towards daily experience sampling have been advocated recently (Hofmann, Baumeister, Förster, \& Vohs, 2012; Thai

\footnotetext{
${ }^{1}$ Some psychologists seem to argue that these problems primarily occur in what they have called "social priming" (Lakens, 2014; for a criticism of the term, see Cesario \& Jonas, 2014), however problems of reproducibility appear to be widespread in psychology (and beyond; Ioannidis, 2005; Yarkoni \& Westfall, 2017).
} 
\& Page-Gould, 2017). However, our approach - Mobile social physiology - takes the premise that human social behavior and cognition heavily rely on the social environment as extension of one's own "head" (Smith \& Semin, 2004) as our starting point. In addition, it takes as a starting point that we observe patterns in human behavior first, before we start predicting mechanisms.

Mobile social physiology uses intelligent technologies like wearable sensors, actuators, and modern analytical methods. This approach will allow us to more accurately pinpoint social (and often non-conscious) processes in real life and likely negate many of our previous theoretical principles. Further, mobile social physiological data collection may uncover more complex models of social behavior than what has previously been found in the lab. This will largely be due to the ability to study patterns in vivo, shifting the focus from the lab to hardly examined dynamics of homeostatic regulation and complex cognitive patterns in real-life relationships. ${ }^{2}$ Adding mobile social physiology to existing experience sampling methods can therefore reveal many hidden treasures of relationship dynamics that are likely predicated on physiological dynamics. In outlining our position, we presume that social processes are embodied (Beckes, IJzerman, \& Tops, 2015) and distributed or transactive (Finkel, Fitzsimons, \& vanDellen, 2015).

To understand these starting points, we rely on the principles related to ontogenetically and phylogenetically ancient processes (sharing of body heat and vigilance processing). These processes position a relationship's core aim as the (co)-regulation and

\footnotetext{
${ }^{2}$ Notably, we explicitly recognize that the processes we study now and that we point to here are made possible by advances in modern technologies. Twenty or thirty years ago, researchers did not have the availability of more advanced statistical programs that provide the analyses with the click of a button, nor did they have the availability of modern sensors and actuators where we can measure and manipulate human peripheral skin temperature with incredible precision. Finally, and importantly, psychological science has also seen a "revolution 2.0" where online frameworks like the Open Science Framework allow for easy data and code sharing and/or easy distinguishing between exploratory and confirmatory findings (through for example pre-registration; see also Spellman, 2015). All these developments put us at an unfair advantage when judging researchers from the past.
} 
reduction of "allostatic load"3. Those ontogenetically and phylogenetically ancient functions are the foundation for higher order functions and provide a framework to study nearly all kinds of relationships (Mandler, 1992). The basis is co-regulation: We think that more complex relationships can then be detected and integrated into this framework to regulate allostatic load from a "predictive coding" account, allowing us to understand how modern social relationships still help us to regain homeostasis (Friston \& Kiebel, 2009).

The goal and contribution of this article is threefold. First, we aim to extend a theoretical model laid out by Beckes et al. (2015) on interpersonal relationships. We start with describing the state of the art of relationship science and further explain the coregulation perspective that we see as unifying for the field. Second, we summarize and provide modern methods developed to support the investigation of interpersonal relationships by extending an approach described by IJzerman, Heine, Nagel, \& Pronk (2017). To illustrate how our approach can be applied to nearly all relationships, we provide examples from our own research with the emphasis on: Caregiver-infant, romantic partners, and therapist-client relationships. We explain how modern analytical methods borrowed from artificial intelligence can help us predict and then develop formal theories that should stand the test of time.

Finally, and crucially, this article presents an integrated app, the Bio-app for Bonding, where we cover modules from four different initiatives (measuring electrodermal activity, peripheral temperature, infant crying, and self-report). The app is posted on GitHub (https://github.com/co-relab/bioapp), where we share the code and keep developing our app. As we relied on our proposed theoretical principles of relationships, we built modules of applications (apps) to study, understand, and intervene (in) relationships in real life. We

\footnotetext{
${ }^{3}$ Allostatic load refers to the overstimulation or understimulation of allostatic systems, which can be caused by "(1) frequent activation of allostatic systems; (2) failure to shut off allostatic activity after stress; (3) inadequate response of allostatic systems leading to elevated activity of other, normally counter-regulated allostatic systems after stress" (p. 37-39, McEwen, 1998).
} 
describe the integration, the different modules, the modern analytical methods used to create these modules, and we explicate how these methods can be used to quickly advance mobile social physiological research and its application.

\section{Theoretical Framework: extending the model of Beckes et al. (2015)}

\subsection{State of the Art in Relationship Research}

In the past few decades, interpersonal relationship research has become one of the core areas in social psychology, providing a rich and fruitful field of discovery. Despite its richness, our understanding of relationships has been overly reliant on theories developed in the socially isolated context of the lab. Therefore, theories of relationships likely have become over- and underspecified. One prominent example of overspecifying is the concept of the internal (working) model, which is typically used to understand how people predict the behaviors of others. The concept of the internal model has become the holy grail of relationship research. ${ }^{4}$

Psychologists often assume that people have an internal, mental (and amodal) representation of others that helps them predict how to act towards other people. The brief summary of how an internal model is ostensibly formed is that people experience a situation (e.g., a loving and emotionally responsive parent) for which they form associative links in memory (e.g., when I am sad, my parent will hug me). They then apply this internal model later in life to know how to act in a novel situation (e.g., hugging a sad partner) when the relevant information is "primed" (e.g., through the sad face of the partner). As the internal model is seemingly ungrounded in aspects like perception, it has been extended to understand nearly all relationships behaviors, which in turn have mostly been understood as consisting of higher order processes, like responsiveness in close relationships (Reis \& Gable, 2015), the

\footnotetext{
${ }^{4}$ The working model can take various forms. Sometimes for example the working model is referred to as "cognitive interdependence". The idea remains the same: People form internal mental representations of others that can be activated through "primes" and can thereafter be acted upon.
} 
role of relationship commitment in its maintenance (Rusbult, Olsen, Davis, \& Hannon, 2004), and accommodation and/or forgiveness in close relationships (Agnew et al., 1998).

Relationship quality, forgiveness, commitment, responsiveness, and accommodation can all be categorized under something that has become known as cognitive interdependence. ${ }^{5}$ One example sequence of steps that is expected to occur from this internal working model's perspective in terms of processing information is: Prime of sad partner $\rightarrow$ activation of (earlier) memory of responsive parent $\rightarrow$ supportive response $\rightarrow$ downregulation of sadness by partner.

This priming perspective has not been without criticism, with a considerable number of failed replications (see e.g., Chueng et al., 2016, LeBel \& Campbell, 2013). As but one prominent example, a large-scale lab study seeking to replicate the sequence that priming "commitment in a relationship" will result in greater forgiveness of partner (Finkel et al., 2002) was not successful (Cheung et al., 2016). In the data from this replication attempt, Finkel (2016) did detect a correlation between a feeling of commitment and a greater tendency to forgive the partner. We infer that this effect is derived from what the person experiences in one's real world relationship rather than it being temporarily boosted through priming. We conclude that forgiveness and commitment are overall related, but that the priming sequence - "activating” greater forgiveness of partner and temporarily boosting commitment - is not very probable and we presume that it was originally detected due to fitting an overly complex model, which was not predictive of the actual psychological processes, to the data.

Beyond these failed replications, the assumption that such a "social priming" sequence can be initiated through an "internal working model" has for long been criticized on

\footnotetext{
${ }^{5}$ The application of such higher order cognitive representations is probably rooted in a longer tradition that points to cognition as being "in the head" and relying on amodal representations. Central to this idea is the metaphor of the mind working as a computer, in which people perceive external stimuli and translate them to a "computerlike" language (like " 0 " and " 1 "”'s; for a criticism of this perspective, see e.g., Barsalou, 1999, 2008).
} 
theoretical grounds (e.g., Beckes et al., 2015; Fiske, 2002). Instead of deriving a string of internal mental processes, Beckes and colleagues (2015) suggested that such action sequences in close relationships first and foremost rely on perception-action mechanisms, perhaps akin to approaches that have become known as "automaticity". Yet automaticity principles depart from the principle that perceivers lack awareness of the process, control, and intentionality, and are efficient in their behavior (Bargh, 1994). For example, people are thought to automatically and unconsciously mimic each other's emotional expressions (Chartrand \& Bargh, 1999). In the automaticity literature, mimicking has been considered to serve as "glue" so that the mimicked person is more likely to affiliate. Like the concepts focusing on cognitive interdependence, the automaticity literature depends on habits and internal representations to facilitate interpersonal relations.

Contradictory, other research has shown that "automatic behavior" is not so automatic, not necessarily mimicked, and instead more context sensitive (Andersen, Moskowitz, Blair, \& Nosek, 2007; see also Fiedler, Bluemke, \& Unkelbach, 2009). For example, people in high (and not low) quality relationships do not mimic their angry partner, but instead respond with an affiliative smile (Häfner \& IJzerman, 2011; Likowski, Mühlberger, Seibt, Pauli, \& Weyers, 2008). Similarly, in high quality relationships people increase their peripheral temperature when they notice their partner's or child's stress reaction (IJzerman, Coan, et al., 2015; Vuorenkoski, Wasz-Höckert, Koivisto, \& Lind, 1969), even though stress reactions typically induce a decrease in peripheral temperature (Vinkers et al., 2013). People's behaviors are typically in service of relationship maintenance. Further, the importance of "habits" (like relationship quality) in such behaviors suggests that temporarily "primed" changes in commitment, attachment, and relationship quality appear nigh impossible. 
To sum up, the "traditional" perspective is that people behave automatically and unconsciously in interpersonal relationships, which rely on "priming" sequences of internal models (e.g., facial expression $\rightarrow$ internal model $\rightarrow$ action); be it conscious or not. We contrast this with a view that homeostatic regulation is the core goal of relationships, relying on direct perception-action sequences initiated upon detecting the partner's emotions. ${ }^{6}$ From this perspective, higher order mental constructs of responsiveness are difficult to prime, and instead habitually formed through frequent and dynamic activations that are first and foremost reliant on maintaining homeostasis. People, having formed a habit focused on being supported by the partner (or by a caregiver), respond supportively towards the partner upon noticing the partner's face, which calls upon basic perceptual mechanisms. They may respond displaying a spontaneous smile to the partner's angry face and/or they may increase one's skin temperature to the other's sad face. The question remains: What are the underlying psychological and/or physiological processes and how can it be that people habituate in becoming responsive to others? The ideas supporting this view are emergent, to date still understudied, and not yet unified. Little empirical data (and surely little finegrained, real-life physiological data) is available. By providing a framework and a methodological approach here, we hope to stimulate data collection to support (or reject) our model.

There are comparable processes found in many endothermic animals (see Ebensperger, 2001; IJzerman, Coan, et al., 2015; for reviews). To further outline the principles behind these mechanisms we turn to the concept of co-regulation (one partner's adaptation to the other partner; Butler \& Randall, 2013; Gottman \& Levenson, 1992). Using

\footnotetext{
${ }^{6}$ This does not implicate that mental representations play no part in interpersonal relationships. Instead, mental representations modulate responses, dependent on longer-term individual experiences throughout one's (relationship) development. One example is the concept of the internal model of attachment. From birth on, infants form a "model" that captures the reliability of the caregiver. This model has been found to be a relatively stable individual difference factor that predicts how people act in relationships (for a review, see Beckes et al., 2015).
} 
co-regulation and principles related to homeostasis (e.g., an infant cry when distressed or cold), we will further clarify our position, partly relying on literature on animals other than humans.

\subsection{Unifying Diverging Theoretical Perspectives in Relationship Research: Co-}

\section{Regulation}

Co-regulation is likely one of the core concepts to unify diverging perspectives in relationship science. It can be defined as "the process by which relationship partners form a dyadic emotional system involving an oscillating pattern of affective arousal and dampening that dynamically maintains an optimal emotional state" (p. 202, Butler \& Randall, 2013). By co-regulating, the dyad can balance allostatic load in more efficient ways. The concept of coregulation can help us understand when and why responsiveness and/or commitment to a partner becomes habituated and/or becomes functional. The example of the smile to a partner's angry face or the skin temperature increase on noticing the partner's or child's stress are all indicative of some core perception-action principles when co-regulating (for a difference between mimicking - as in the case of negative reciprocity - and co-regulation, see Figures 1A \& 1B).

Although initially the idea of co-regulation was framed from a perspective comparable to the internal model and was construed as conscious emotion regulation patterns towards one's partner (Gottman \& Levenson, 1992), the understanding of co-regulation quickly shifted to the inclusion of lower order, less conscious mechanisms with attention to perception-action links. In contrast to the static "social priming" models in which forgiveness, commitment, or even self-control can be briefly activated, the co-regulatory approach assumes a more complex "bi-directional statistical dependence between relationship partners over time” (p. 203, Butler \& Randall, 2013; for an example of bi-directional 
statistical dependence related to the buffering of stress, see Figure 1 and IJzerman, Heine, et al., 2017).

This bi-directional dependence relies on principles of providing allostatic balance. Allostatic balance is a broader concept than homeostasis, since it includes a "coordinated variation of psychological and biological systems that optimize performance and minimize costs" (p. 204, Butler \& Randall, 2013; Sterling, 2003). Vital to allostatic balance is the regulation of stress, which in its turn depends on pressing environmental demands (like temperature changes). These demands, evolutionarily, have hinged on a need for (and thereafter provision of) safety and security, and, in extension, the ability to explore (Bowlby, 1969). Although most modern perspectives in attachment theory focus on subjective experiences of safety, Bowlby's (1969) original departure point was that relationships are organized around "control systems" that let infants attend to their needs and call for care from the caregiver, who in turn provides the infant with safety and security from danger, coldness, and around the provision of food. Only recently, the importance of the co-regulation of these biological urges was brought back into consideration, with relationship partners or parents providing reduction of vigilance to stressors in the environment (Beckes \& Coan, 2011) or helping with the regulation of body heat (IJzerman \& Hogerzeil, 2017).

Co-regulation and the achievement of allostatic balance thus hinge on safety and security. For endothermic animals, safety and security relies first and foremost on being safe from predators and from the cold. Take for example ostriches. When they need to eat, they are faced with two challenges: (1) they need to keep out a watchful eye for predators and (2) they need to pick up food from the ground with their long necks. On their own, the rate of picking up food is low. Yet, when other ostriches are around, the rate of eating goes up, because looking out for predators is distributed over the group. This distribution increases the amount of food the ostrich can take in and decreases the individual cost of vigilance 
processing (Krebs, Davies, \& Parr, 1993). Adult humans have a similar vigilance processing distribution. Coan, Schaefer, and Davidson (2006) have found that when the participant's hands are being held during an electric shock, the activation of brain areas related to stress is reduced to a minimum. This is not true for the participants who are alone. These effects are more pronounced when the hand holder is someone familiar (e.g., a significant other) and/or the relationship quality is higher. The central idea is that - like ostriches - threat (and thus stress) becomes distributed over the dyad through a process of co-regulation.

A second example from the animal literature is about a type of rodent called the Octodon Degus. The Octodon Degus, when housed together with other animals (in an environment of 15 degrees Celsius), retains a higher peripheral temperature and lower basic metabolic rate compared to when being housed alone. In rodents, social thermoregulation has thought to be one of the most important motivating forces behind group living, especially when temperatures drop (Ebensperger, 2001). For humans, drops in peripheral temperature (absent other beings) also occur to preserve heat in the core. Human infants, for example, need others to help regulate their temperatures (their bodies are too small to do this by themselves). In one study, when a mother left the room in the well-known Strange Situation, infants' peripheral temperature decreased and the temperature only returned to baseline once the mother returns (Mizukami, Kobayashi, Ishii, \& Iwata, 1990).

Distribution of temperature and of risks are formative for interpersonal relationships in their simplest form. They later become "sociometers" as they become habituated into more stable relationship orientations. Recent, unpublished, findings for example show people's desire to socially thermoregulate is robustly related to attachment avoidance (Vergara, Hernández, Juame-Guazzini, Lindenberg, Klein, \& IJzerman, 2018). Further, people also relate trustworthiness of inanimate objects (like consumer brands) to temperature, as more 
trustworthy brands elicit higher temperature perceptions (IJzerman, Janssen, \& Coan, 2015; see also Clark, 2013).

Central to bonding is thus the degree to which the infant's needs of stress regulation and temperature can be outsourced, or co-regulated. One of the hallmarks of attachment theory is that the early experience of safety, security, and the ability to explore becomes a stable individual pattern to predict the behavior of others later in life. Attachment is also thought to form the basis for interaction styles in adult romantic relationships (Fraley, Waller, \& Brennan, 2000), in group participation (Smith, Murphy, \& Coats, 1999), in therapy (Mallinckrodt, Gantt, \& Coble, 1995), and even in the "interaction" with deities (Kirkpatrick, 1998).

To summarize our theoretical framework, there are a few core principles when it comes to studying relationships. One of the starting principles is that we co-regulate. Second, co-regulatory processes primarily revolve around the distribution of vigilance processing and thermoregulation. Third, stable individual difference patterns in bonding and attachment develop, and these relate to individual differences in vigilance processing and thermoregulation (Vergara et al., 2018). Fourth, cognitively more complex dynamics (like forgiveness, commitment, and conscious forms of emotion regulation) are likely "built" on top of lower order co-regulation patterns (Mandler, 1992). We have now defined the basic principles. However, concepts like bi-directional statistical dependence and allostasis are still too vague for a coherent theoretical model. How can one reliably study these to formalize the concepts?

\section{Our Approach: Mobile Social Physiology}

\subsection{From the Lab to Mobile Social Physiology}

Measurements of feelings of safety from physical threats and from decreasing temperatures are crucial to studying elementary forms of co-regulation. More precise 
interpretations of basic co-regulatory patterns and how they relate to our more complex emotional lives can be discovered by iteratively adding stable individual differences (e.g.., attachment), adding measurements related to understanding of the context and meaning (e.g., self-reported experiences of discrete emotions), and/or the measurements pertaining to subjective perceptions of the relationship and other higher-order cognitive dynamics (e.g., daily perceptions of responsiveness to/of and/or commitment of/to the partner).

Modern technology allows us to engage in this iterative process through the application of sensor technologies in real life settings. But how? What kind of hardware is needed? And what are the reasons to prioritize one type of hardware over another? Based on our experience with this kind of research, we see it as essential that these technologies are wearable, wireless, unobtrusive, and ubiquitous. Furthermore, it is desirable to - a priori - set the goals of the research to make progress as quickly (and as reproducible) as possible. We won't detail ways to develop a research workflow or how to deidentify data, as this is addressed elsewhere (Joel, Eastwick, \& Finkel, 2018; Klein, Hardwicke, et al., 2018). Instead, we will outline basic choices for creating the system to research co-regulation and provide examples for applying and integrating these methods to study vigilance processing and sharing of body heat.

\subsection{Developing mobile social physiology systems: The hardware and software}

The first choice in the development of mobile social physiology systems is deciding on the kind of external device to use. Beyond accuracy of measurement, an external device can offer (at least) two advantages: (1) temporary storage and (2) visualization. Most external devices offer internal storage. However, storage on most devices is not sufficient to store data continuously measured for weeks or even months and it is impractical to do frequent readouts. Smartphones and tablets become more and more integrated in our daily lifestyle and are rapidly developing to offer internal memory and visualizations with capabilities 
sufficient for our requirements, and can thus offer a reasonable alternative for a PC in the lab if they are combined with external devices like sensors. At the time of developing our systems, wearables (like smartwatches) were not yet offering the capabilities of storage and visualization required for our studies. Due to their rapid development, we believe that they combined with sensors - are the future for mobile social physiology.

\subsubsection{Hardware}

We tried to rely on external devices that are relatively non-intrusive. For example, in our approach to study the distribution of vigilance processing, we considered equipment from Mind Media and Thought Technology, which offer units that can connect and stream or log data simultaneously from several sensors (e.g. electrocardiography (ECG), electromyography (EMG), and skin conductance (SC)). These units require wired connections to external devices and to gel electrodes, thereby limiting the participant's freedom of movement. A second disadvantage of using base units like Mind Media or Thought Technology is that they collect data using gel electrodes, which are still the gold standard within physiological sensing. Gel electrodes are intrusive, as they need to be replaced every few hours and their placement can be obtrusive (e.g., require placement on the palm, two fingers of the hand or sole of the foot for SC measurement), thereby limiting the user's freedom of movement.

We then considered sensors, like activity trackers. Activity trackers can be worn for longer periods of time. They are also low cost and often provide immediate feedback on a screen integrated in the wristband. The transition from the lab to the field is only possible however when the quality and the accuracy available in the lab are maintained. Although activity trackers are low cost and offer easily accessible solutions, they are aimed at daily use to gain a healthier lifestyle or to improve a workout. They are typically not developed for scientific research, usually are not very accurate, and typically do not allow access to raw data. We have also explored other types of sensors, like those backed by crowdfunding (e.g., 
the "AngelSensor"). Although crowdfunding often offers promising developments and innovative ideas, they usually underperform against expectations and are simply too unreliable for scientific projects (Tarus, 2016; Alois, 2014). Finally, we also considered a sensor to which we had access to its firmware in the past, but now no longer have it (and thus started using a different sensor and signed an NDA to retain access to the firmware).

The activity trackers and wearables (like smartwatches) did form the basis for our solution; namely the use of smart textiles. Smart textiles have the ability to conduct electricity and have therefore the possibility to replace standard gel electrodes. They can be combined with sensors that are scientifically more reliable and that can be easily integrated in clothing and wearables, therefore feeling like normal products and lowering intrusiveness considerably (see Figure 2B).

\subsubsection{Software}

After deciding on smart textiles as our device of choice, we moved onto software. The first step in software development for mobile social physiology is choosing the operating system of one's external device. Currently three available operating systems dominate the market - Android, iOS and Windows Phone - of which the last is the weakest option since there are only a few smartphones and tablets running on Windows. We consider Android the best option, because it is open-source and most widely used in mobile devices (e.g., smartphones).

After deciding on Android, the next step was our decision for communication method. Wi-Fi, Bluetooth, and Bluetooth Low Energy (BLE) are the most commonly used options. We prefer BlueTooth to Wi-Fi for communication. When needing a constant connection, a change of location could lead to data loss, while Bluetooth offers a possibility to transmit information from the device to the phone directly. We favored BLE over Bluetooth, as BLE is integrated in newer Android devices and is more efficient. When using (university-owned) 
devices (that do not have phone subscription) for storage purposes only, an infrequent Wi-Fi connection is sufficient if data is stored locally on the Android phone (and communicated at least once a day to a central server). Often it is possible to use a combination of BLE (to transfer the information from the device to a phone) and Wi-Fi (to transfer the information from the phone to the server, albeit infrequent). The frequency of transferring data over WiFi depends on the information being acquired, with audio needing a far more frequent connection. We communicate data to our server when the phone's storage is $80 \%$ filled.

Then device-specific software needs to be developed. We strongly believe we should use open-source methods. The benefit of relying on open-source code is that other developers can easily adjust code to the requirements of their study and improve when necessary. Using GitHub allows our code to be open-source, so that developers can check, correct, and build onto our apps. The benefit of using GitHub is the structure they offer (very comparable in its use to the Open Science Framework, a website that has become popular recently amongst psychologists). Other developers can fork our code, and then post versions of their code so that it is easily adapted to their study requirements as an attachment to the original code thereby creating a database of code for related research. Unfortunately, using open source software limits the types of devices that could be used. For example, when we started our research, despite being frequently used, Fitbit could not supply us with open source software.

Finally, we typically deal with participants or clients for whom privacy is crucial. Thus, a platform like Apple Research Kit, where data is stored outside of the European Union (in the United States), is less than ideal. Privacy of participants still remains a concern. Our current solution to protect participants' privacy is by 1) using https on our server, use e-mails and passwords to connect from the app to the server, and generating a private token for the app to send the information. We thus have some basic security that decreases the chances of 
devices being hacked and considerable attention needs to be devoted to move this type of research forward (cf. IJzerman, Heine, et al., 2017).

\subsection{Examples - Recent studies using mobile social physiology}

After outlining our general approach, we outline the specific details of our research across the spectrum of interpersonal relations, ranging from infants with severe disabilities to close relationships to team processes. In our pilot studies on these groups, we commenced testing the different sensors and developed our software. All of the example groups we study can be investigated using the same principles of co-regulation, albeit varying along a continuum of less to more complex social interactions.

\subsubsection{Research Line 1: Visualizing Distribution of Vigilance Processing/Stress}

Distribution of vigilance processing between one person and another can be assessed through physiological correlates of stress, which in lab research has been studied at the level of the brain (Coan et al., 2006), stress hormones (Kirschbaum, Pirke, \& Hellhammer, 1993), and through physiological signal monitoring (Giakoumis, Tzovaras, \& Hassapis, 2013). In earlier work, Schuengel, Sterkenburg, Jeczynski, Janssen, and Jongbloed (2009) reported on the buffering effect of a secure relationship for persons with a severe intellectual disability on their stress levels. In this study of a real life therapeutic setting, the physiological measure of stress was collected with the VU University Ambulatory Monitoring System (VU-AMS: De Geus \& Van Doornen, 1996).

Measuring the distribution of vigilance processing can go from relatively simple (physiological measures) to very complex (inferences of why a person experiences stress in the job context). People have a different range in their capability of processing complexity and predictability in their close relationships, however across the spectrum of intellectual development, the principle in terms of physiological correlates is the same. That is why we 
can apply the same co-regulation principles to people with severe disabilities, like persons with a severe intellectual disability or children with Prader-Willi Syndrome (PWS).

PWS is a neurogenetic syndrome resulting from an absence of expression of paternal genes on the long arm of chromosome 15q11-13 and has a prevalence of approximately 1/15.000 - 1/25.000 births (Cataletto, Angula, Hertz, \& Whitman, 2011; Reus et al., 2011). In newborn infants, PWS is characterized by severe hypotonia, which is expressed in a poor cry, reduced movement, and a weak suck reflex (McCandless, 2011; Reus et al., 2011). The infant's development of more complex social interaction skills that allow others to read, understand, and interpret signals that indicate the infants needs to "outsource" vigilance processing is severely delayed. As the infants are in fact not yet capable of showing stress responses, the goal of these studies is to measure and visualize the child's "need for distributing vigilance processing" so that the parent can more directly and more accurately support his or her child.

The most elementary level of co-regulation between parent and child is the activation of the Autonomic Nervous System (ANS). Since the infant's ANS is not impaired, it helps us understand the distribution of vigilance processing in its most elementary form. Frederiks, Croes, Chen, Bambang Oetomo, and Sterkenburg (2015) sought to visualize ANS activation with the Sense system. The visualization functions as an additional cue for parents to better read, understand, and interpret their infant's signals (for an example of the underlying data for this visualization, see Figure 3).

The design of the Sense system started with defining the most suitable sensor for the aim of the study. Since the visualization of the distribution of vigilance processing is a new area of research, the choice for the sensor type was based on a related field of research: affective computing. In affective computing, ANS activation is measured to detect emotional arousal, and, when measured in dyads, can be understood as the need of the child to distribute 
vigilance processing. Electrodermal activity (EDA) is the standard physiological measure to assess emotional arousal in affective computing research (Picard, Fedor, \& Ayzenberg, 2016) and has therefore been chosen as the sensor type for the Sense system.

The first step in developing our mobile social physiology approach to visualize the distribution of vigilance processing was measurement. In an initial study on children with PWS, the child's general arousal level and responses to environmental stimuli were monitored during the interaction with their mother (Frederiks et al, 2015). A Med-Storm Pain Monitor (Storm, 2012) was chosen as first measurement device due to its high accuracy and reliability. Both this monitor and the VU-AMS system rely on a wired connection to the base module and/or to a laptop PC(See Figure 2A). This connection is not only bothersome for children with PWS and their parents, but also limits their movements.

Due to this previous experience, the Sense system evolved into a wireless system, for which a Shimmer TM 2R BioPhysical sensor module was used. Shimmer offers small, lightweight sensor modules that are able to stream data wirelessly via Bluetooth, store data on an integrated SD card, all the while having long battery life. The sensor data is superior in accuracy and details as compared to regular fitness trackers, as they are collected with gel electrodes. Thus, Shimmer offers a wireless sensor that is according to scientific standards. The ShimmerTM2R BioPhysical sensor module is connected to a smart sock and measures the skin conductance over the wearer's foot (see Figure 4).

The technology used in the smart sock is "smart textile", offering the convenience of wearing the sensor at the location on the body (e.g., wrist, ankle, chest) most suitable for the specific population. We customized the Shimmer sensor to improve the wearer's comfort and to make the system as unobtrusive as possible by using fabric electrodes, integrated in a sock, instead of the standard gel electrodes. The SC sensor sock has two textile electrodes made of Shieldex ${ }^{\circledR}$ textile. Each textile electrode is connected through Shieldex ${ }^{\circledR}$ conductive yarn to a 
snap fastener, which allows for connection with the Shimmer sensor module (in a similar manner as connecting a gel electrode to the Shimmer sensor module via a snap fastener). The Shimmer ${ }^{\mathrm{TM}} 2 \mathrm{R}$ BioPhysical sensor module transfers the skin conductance data via Bluetooth ${ }^{\circledR}$ to a tablet PC. This wireless version of the system was validated in a pilot study where the interaction between an adult with a visual and severe intellectual disability and his caregiver is observed (Sterkenburg et al., 2017). Figure 5 shows the wireless system used during the pilot study.

There are a couple of disadvantages to this system. First, the Shimmer technology can only assess one physiological measure at a time. Shimmer sensors have a main circuit board for the general functionalities (like Bluetooth communication) to which several separate circuit boards, dedicated to one specific sensor modality (e.g., ECG), can be connected (only one at a time). Second, our present version of the system was developed for new-born infants with PWS and therefore optimized for this target group. Although the system is also being evaluated with adults with severe intellectual disabilities, the system can only be used for participants who stay seated. Since the fabric electrodes are located at the sole of the SC sensor sock, walking on them creates noise in the SC signal and thus the system cannot yet be used in most real mobile social physiology contexts. The step to a follow-up version where participants move around is only minimal from here on. It requires the integration of fabric electrodes in a wearable intended for a different body part that offers comparable sensor measurements as the sole of the foot.

\subsubsection{Research Line 2: Temperature Co-Regulation}

Like with the distribution of vigilance processing, we developed sensors and software to measure the distribution of temperature regulation. Such co-regulation of body temperature first and foremost is dependent on sharing body heat, but temperature regulation has extended to already more complex social interactions: Social exclusion for example leads to a lowering 
of peripheral temperature (IJzerman et al., 2012), while a variety of "higher-order" relationship behaviors (like projecting relationships onto inanimate objects; IJzerman, Coan, et al., 2015) have been linked to social thermoregulation (for an overview on cothermoregulation, see IJzerman, Heine, et al., 2017). Some early research has shown, for example, that mothers' peripheral temperature rises when they hear their infant cry (Vuorenkoski et al., 1969).

In some of our early research on peripheral temperature, we used a DS1810 1-wire digital thermometer that is typically used for measurement in industrial coolers, measuring 1/32 of a degree accuracy between -55-125 degrees Celsius. This sensor was connected via a wire to a computer. The computer in turn was connected with a LAN cable to a wireless server where the data was stored, allowing us to detect a 0.39-degree Celsius decrease after social exclusion in 500 seconds (IJzerman et al., 2012). Despite its accuracy, this sensor is like the VU-AMS and the Med-Storm Pain Monitor - burdensome, as it relies on a wire attached to the computer. This is of course even more burdensome when using it outside the laboratory and/or with more sensitive populations (like mother-infant dyads).

A more mobile solution that is often considered the gold standard for thermophysiology research is the "iButton" (Van Marken-Lichtenbelt et al., 2006; see Figure 6), which is a small button that can be attached to the body (typically the finger) with a sticker. In social psychology, the iButton has been used, for example, to investigate the link between detecting deception and changes in peripheral temperature (van 't Veer, Gallucci, Stel, \& van Beest, 2015). There are two potential objections to the iButton for broader adoption. First, when using it in research, the button itself takes a considerable time before it warms to the participants' skin, thus providing considerable noise in measurement. Second, when using the button outside of the lab on the finger it is too intrusive, because it hinders the participants' daily functioning. In addition, when applying to the skin, the button easily 
detaches from the body in daily use. We also explored options to measure temperature supported by crowdfunding (like the "Angelsensor") but these initiatives did not develop to full maturity or did not receive enough backing (Comstock, 2016). We also considered the BlueMaestro Tempodisc, as we initially had access to their firmware. Because we lost access to their firmware we decided to switch to the MyTemp sensor, which can be more easily attached to the index finger (Figure 7). Its current sensor has a bias $<0.1$ degrees Celsius (although it can only measure every 5 seconds; Bongers, Hopman, \& Eijsvogels, 2017). Although the current solution is not yet used for commercial purposes, it is adequate for our research requirements.

\subsection{Analytical Approaches to Deal with Complexity in Predictive Coding and Free Energy Principles}

So far, we have been discussing real time data collection with modern technologies. However, data analysis plays an equally important role in developing accurate causal models of human behavior and cognition. Concepts like bi-directional dependence and co-regulation are still sufficiently vague. However, previous approaches have not fared much better, as is evidenced by the many failed replications in psychology. Studies conducted during past years by "the replicability movement" have clarified that it is hard to replicate prior scientific work (with some estimates of studies replicated as low as 39\%; Open Science Collaboration, 2015). Some have argued that the problem is particularly potent in the "social priming" domain (Lakens, 2014), although replication efforts show that the problem is applicable to the entire domain of psychology (and beyond). We have pointed to the problem of underspecified theoretical predictions, one that will worsen if it's unguided by overarching principles. In the first section of this article, we have outlined our overarching theoretical principles. Here we outline how our principles can be turned into formal theoretical models 
through data-driven methods, which includes pinpointing individual differences in coregulatory patterns.

Psychologists have typically focused on studying complex, mechanistic explanations in small (underpowered) samples and have combined this with publication bias and with fitting models to data a posteriori. This has resulted in something called procedural overfitting. Overfitting happens when data are modeled to a specific dataset in an overly complex way. The (ad hoc created) hypothesis is then unlikely to be replicated in another dataset (see also Gelman \& Loken, 2013), causing implausible models to outperform unbiased models (cf. Yarkoni \& Westfall, 2017), such as in the case of "priming commitment in a relationship" (Finkel, 2016). At the same time, researchers have "underfitted" their theoretical models, by applying models to data that are too simplistic, such as in the case of automatic mimicking of conversation partners (Bargh, 1994).

It is clear that social psychological science has a replication problem. Perhaps in relationship literature the problems are less formidable, because of a focus on convergence of methods. We suppose that the relationship literature typically investigates the right variables (such as commitment, accommodation, self-control, and so forth), but that the exact relationships between constructs are underspecified. In relationship studies, researchers are probably "hitting" the right variables (e.g., commitment) which results in relatively low bias, but they produce too much noise at the individual study level. This means that, as a result, predictions for new studies are not sufficiently precise (as in the priming of commitment example). Ultimately, as a field, we want to reduce both bias and noise, so that we can predict under which conditions phenomena occur and when they don't (see Figure 8 from Yarkoni \& Westfall, 2017, for an illustration).

One way to reduce bias is to use an appropriate model (one that fits to the true function and is not too simple). However, since the true function is usually unknown, 
regularization is used to avoid overfitting. Fitting a function that is not underspecified and adopting regularization is rarely used in social psychological science. As one example, IJzerman, Čolić, et al. (2017) recently used supervised machine learning to falsify a proposition asserted by Van Lange, Rinderu, and Bushman (2017). They suggested that the distance from the equator predicts people's self-control, a proposition which was shown to be underspecified and thus rejected by IJzerman, Čolić, et al.’s (2017) supervised machine learning analyses (indicating that equator distance could be considered a negligible predictor of self-control, essentially indistinguishable from random noise).

IJzerman, Lindenberg, et al. (2018) used a comparable approach to identify the most important predictors of core body temperature in a first phase, followed by a path model in a second phase where they used cross-validation (showing that distance from the equator predicts social integration, which in turn predicts (and protects) core body temperature). We envision that in the first phase of the mobile social physiology approach we have advocated here, comparable supervised machine learning approaches can be relied on, for example, to identify which types of co-regulatory patterns of peripheral temperature are most indicative of high-quality relationships (a script and explanation to apply machine learning can be downloaded from IJzerman, Pollet, Kun, \& Ebersole, 2017: https://osf.io/x53pu/).

Patterns should thus be observed from experience sampling data using our sensors and one can do so using basic machine learning. In a second phase and in an attempt to understand more complex interrelationships between different aspects of co-regulation (of temperature and stress) and predictive coding (via responsiveness and relationship commitment) we envision a focus on deep learning using convolutional neural networks, in ways comparable to the approach of the crying detection that will be discussed in section $3.3)$.

\section{The Bio-App for Bonding (BAB)}


Our approach will dramatically reduce the gap between theory, data, and application. In fact, we provided a couple of examples of recent studies that used the mobile social physiology approach. In these studies, four separate application modules were created for the specific aim of that study. Each module will be described in detail below. All of the measurement modules mentioned below are now programmed into one app, our Bio-App for Bonding (BAB), available on GitHub: https://github.com/co-relab/bioapp.

\subsection{Measurement Module 1: The Flower Module}

For the parents of infants with PWS we have developed a signal so that parents can more easily interpret their infant's subtle communication behavior. The original goal of the Sense system was to visualize the infant's need to distribute vigilance processing to the caregiver. For the Sense system, we adapted Shimmer's app from a graphical representation of the sensor data to a visual representation in the form of a flower that can communicate the infant's stress to the caregiver.

This visual element represents the skin conductance signal, which consists of two elements, a tonic element and a phasic element. ${ }^{7}$ In the Flower app, the tonic element is extracted from the raw skin conductance data (as received from the Shimmer sensor) with the use of a $1 \mathrm{~Hz}$ low-pass filter. This tonic element is then visualized in a green flower that changes in size correspondingly to the alterations in the measured skin conductance level. The radius of the flower is determined by dividing the tonic value by a constant. This radius has a lower and an upper threshold (meaning that the flower is not able to reflect alterations below or above a certain tonic value) to make sure the flower remains visible in the screen.

The phasic element is extracted using a high-pass filter on the tonic value. To calculate the number of skin conductance responses (SCR) per second, each phasic value is

\footnotetext{
${ }^{7}$ The tonic element, also referred to as skin conductance level (SCL), is a slow varying element, which indicates the overall level of sympathetic arousal (Benedek \& Kaernbach, 2010a,b). The fast-varying phasic element shows a steep increase and a slow recovery of skin conductance (Benedek \& Kaernbach, 2010a,b). The phasic element is also referred to as "skin conductance response" (SCR) (Benedek \& Kaernbach, 2010b; Ogorevc, Geršak, Novak, \& Drnovšek, 2013). SCR indicates stimulus-specific responses or non-specific responses (e.g. a person's thoughts) (Benedek \& Kaernbach, 2010b).
} 
compared with the previous phasic value; if the difference exceeds a set threshold, the change is registered as a peak and all peaks in a period of 1 second are summed. These peaks are visualized in the flower through additional (orange) petals appearing when peaks are detected. If the number of peaks exceeds a threshold, the opacity of the orange petals is gradually increased (thus making the petals visible). When the number of peaks drops below the threshold, the opacity is gradually decreased and the petals fade out. The SCRs are thus reflected in the occurrence of orange petals (see Figure 9). As such, the signal from the child is translated so that a parent can interpret the child's needs in relation to "distribution of vigilance processing".

At present, the algorithm for the flower is still based on a linear and relatively simple perspective. As we progress, we anticipate creating easy to use visualizations based on machine learning - and especially deep learning - to visualize relationships between stress, temperature, attachment, and other relationship-related constructs. These visualized relationships can be used in relationships between therapist and client, mother and infant, and managers and their teams to further understand and optimize interpersonal relationships. Notably, for those less inclined to data science, it is always possible to submit datasets to socalled "Kaggle" competitions, so that expert data analysts can compete to create the most plausible model from the data.

\subsection{Measurement Module 2: The Temperature Module}

For the temperature co-regulation research approach, we selected MyTemp temperature sensors. In our first iteration, we used a Blue Maestro TempoDisc, for which we developed the app called Closeness, via which we communicated the temperature data from the sensor via Bluetooth Low Energy to an Android phone, and from the Android phone to our server (Van Emden, 2015). We reprogrammed this app to communicate with the MyTemp sensor. Crucial to being able to infer co-regulation is accurate timing for two 
different sensors. We conducted first tests with the app to measure individual thermoregulation in tasks where teams debated each other. In order to know whether participants were close together, we had different sensors communicate via one app. However, after the first tests with the app, it was clear that one Android phone could not communicate information from multiple sensors, as it rendered our temperature reading inaccurate. We then updated our app to dedicate one Android phone to one sensor and tested its temperature reading (IJzerman, Ligtenberg, \& Verbeke, 2017). In addition, we adapted this app for future use, so that server information (for other users) and participant information could be easily updated on the app itself. Furthermore, we now updated the temperature portion of the app so that when using the app for research, one can enter two different mac addresses, one for the person being measured and one for the partner. The app then automatically recognizes when the partner is within 4 meters and adds a 1 to the database when the partner is close and a 0 when the partner is further than 4 meters. Finally, one of the goals of the app was to relatively easily integrate information from other sources, such as experience sampling via questionnaires or via cues other than peripheral temperature, like infant crying, to which we turn to next.

\subsection{Measurement Module 3: The Crying Module}

Co-regulation likely depends on a complex interrelated set of cues: The infant needs to cue the caregiver that it is cold and needs to warm up, and - like in other animals - crying is one way to signal this need (Awam, Catana, \& Mortola, 2011). Parents of course do not mimic this cry; instead, they provide complementary responses to sooth the infant. Studying this pattern in greater detail requires being able to infer connections between the crying of the infant and the skin-warming response of the caregiver. Inferring infant cries requires a detection algorithm so that the cry can be related with thermal data. Creating such algorithms is crucial for accurately representing research on co-regulation. 
In our first step to model co-regulation between mother and infant, we focused on accurately capturing the infant cry by making a simple audio recorder that can be used in any smartphone. However, this feature by itself was not sufficient: keeping full recordings, around the clock, and manually locating the relevant time intervals is time-consuming, tedious, and requires prohibitive storage capacity. Adding just the recording feature to our design would thus entirely prevent our mobile social physiology solution.

Therefore, in the second step we created our smarter solution that fits with the issue we alluded to before in regards to accuracy of data processing: An automatic baby cry detector in our Android application. The goal of the application is to process, in real time, audio data obtained from the phone's microphone, and to look for onsets of a baby cry. Only the audio segments where a baby cry is spotted are kept, together with the corresponding time markers, thus greatly reducing the volumes of data to store and process. The main challenge is to perform cry detection in real time, while maintaining high accuracy. The application consists of several components and performs the following steps (see also Figure 10):

- Record audio from the environment using the phone's microphone.

- Analyze the recorded acoustic signal in real-time to detect segments that contain instances of a baby's cry.

- Store only these segments and their corresponding timestamps (for correlation with thermal data).

The data for the algorithm consisted of several tens of hours of audio recordings made by parents with babies in the Netherlands (Lavner, Cohen, Ruinskiy, \& IJzerman, 2016). These recordings contained various sounds, like crying, parents talking, doors opening, and so forth. The first step was to annotate these events (which we did using two hours of recordings, down to the millisecond), with more 
than 50 different event types, and a total of several tens of cry events. The cry signal ${ }^{8}$ is characterized by high fundamental frequency (250-600 Hz, Várallyay, 2007, 2009), and a harmonic structure (see Figure 11). These characteristics determine the parameters used by our baby cry detection application. We then tested two different supervised learning algorithms for baby cry detection: one based on a relatively simple classifier, and the other based on a novel deep learning approach.

Exploratory approaches like machine learning have been advocated in the social sciences to reduce false positives and to pinpoint patterns of variables more accurately. Such approaches use algorithms to iteratively discover patterns in data (e.g., IJzerman, Pollet, Ebersole, \& Kun, 2017; Yarkoni \& Westfall, 2017). In the rest of this section, we describe two machine-learning algorithms to classify infant cries, compare their performance, discuss the motivation behind selecting one of them for our application, and show their relevance for this type of research. The first algorithm is based on a regularized logistic regression classifier (Deng, Sun, Chang, \& Han, 2015) and consists of the following steps:

a. Pre-processing and feature extraction. The audio signal was divided into sections of 100 milliseconds and a set of audio features, known to distinguish between different types of audio signals, was computed from each segment. The features include the Mel-Frequency Cepstrum coefficients (Quatieri, 2002), the fundamental frequency of the signal (pitch), harmonicity factor (Cohen \& Lavner, 2012), harmonic-to-average power ratio (Cohen \& Lavner, 2012), short-time energy, and zero-crossing rate. These features provide temporal and frequency measures that are useful for the detection of cry signals. One feature vector, consisting of the above features, was computed for each segment.

\footnotetext{
${ }^{8} \mathrm{~A}$ baby cry is a voiced signal, which means that its production involves periodic movements of the vocal folds, produced by a rapid flow of air through the larynx. This in turn generates a periodic excitation which is transferred through the vocal cords and creates the cry sound.
} 
b. Training and testing of the algorithm. In order to form a model, generally one has to divide the data into two sets: A training set and a test set. The training set in our case contained the first part (several minutes) of the audio recording, where the remaining comprised the test set. In the training procedure, a probabilistic model for distinguishing the infant cry from other sounds is formed using regularized logistic regression ${ }^{9}$. We could then validate this modelin the test set.

c. Test. After we formed the model of the infant cry, a crucial step for the evaluation of exploratory research is to establish detection rate versus false-positive rate. ${ }^{10}$ Since our aim in the algorithm was to obtain enough data for examining the co-regulatory patterns between a baby and a caregiver using cry events as a primary variable to predict attachment, the importance of detecting many baby cry events is obvious. However, a low false-positive rate is perhaps more important, to avoid mixing the data with nonrelated events, which may hinder meaningful conclusions (and record noises that are privacy-sensitive). Therefore, we focused on the trade-off between the false-positive rate and the detection rate, and aimed to lower the false-positive rate, even at the expense of the detection rate.

The second algorithm we tested was based on deep learning (Bishop, 2006). Deep learning algorithms are usually implemented using convolutional neural networks (CNNs) and utilize multiple neural network layers to extract features from multiscale representations of data (Goodfellow, Bengio, \& Courville, 2016). When a suitable set of features is a priori

\footnotetext{
9 The logistic regression can be viewed as a hypothesis function $h_{\theta}(x)$, which approximates the probability that a given audio segment i contains a baby cry, corresponding to an input feature vector derived from that segment $x^{(i)}$. For the hypothesis function a sigmoid (logistic) function is used, where $h_{\theta}(x)=1 /\left(1+\mathrm{e}^{-\theta^{T} x}\right)$, and $\theta$ is a vector of weighting parameters. Given the training dataset with the feature vectors and their corresponding labels, and a pre-defined error function, the parameters are set to minimize the error function using a gradient descent algorithm.

${ }^{10}$ The detection rate (also known as sensitivity or recall) is defined as the ratio between the number of true-positive events (i.e., the number of cry events correctly identified [true positives]) and the total number of cry events in the recording set [true positives + false negatives]. The false-positive (or false-alarm) rate is defined as the ratio between the number of false positives (non-cry events identified erroneously as cry events) and the total number of non-cry events in the recording set (true negatives + false positives).
} 
unknown, deep learning algorithms can have major advantages over traditional machine learning algorithms. They have wide applications in the fields of computer vision, natural language and speech processing, amongst many others. ${ }^{11}$

To use a CNN-based classifier, we first represented our audio signals as twodimensional "images", for which CNNs are better suited. ${ }^{12}$ In this representation, the horizontal axis of the "image" corresponds to time and the vertical axis corresponds to frequency (for an example, see Figure 12). In our implementation, we constructed a CNN of 6 layers, which provided a balanced tradeoff between complexity and performance. We again split the data into a training and a test set and used a backpropagation algorithm to train the CNN. ${ }^{13}$

For the evaluation of the quality of detection/classification models we used a Receiver Operator Characteristic (ROC) curve. ${ }^{14}$ A classifier is better than another if it has a higher detection rate and a lower false-positive rate. When comparing the CNN classifier to the logistic regression classifier, we observed that the former performs better for low falsepositive rates (see Figure 13 for the ROC curves). The performance was similar or perhaps even superior for the logistic regression classifier in case of high false-positive rates. The logistic regression classifier yielded a detection rate of $86 \%$ and a false-positive rate of $2.5 \%$, while for the $\mathrm{CNN}$ with the same detection rate a false-positive rate of $1.8 \%$ was obtained. However, to obtain a much lower false-positive rate, we set a detection rate of $50 \%$ for the logistic regression classifier and received a false-positive rate of $0.07 \%$. This low falsepositive rate, along with the fact that the logistic regression classifier is simpler for

\footnotetext{
${ }^{11}$ Though powerful, designing a CNN is a difficult task as the number of parameters is typically very large (from thousands to millions of parameters). This makes the design and implementation of CNNs considerably more computationally intensive compared to traditional machine-learning algorithms. However, the $\mathrm{CNN}$ does not require typically a pre-processing and a feature extraction step.

${ }^{12}$ We chose to use a log Mel-filter bank (LMFB) representation, which is related to Mel-Frequency Cepstrum coefficients (Quatieri, 2002).

${ }^{13}$ Note that in LMFB representation, each pixel represents a frequency range (in logarithmic scale). Cry signals are harmonic signals with a distinctive spectral content that is typically concentrated at the low frequency bands. Thus, we designed the CNN architecture to achieve high frequency resolution at the low frequency bands.

${ }^{14}$ The Receiver Operator Characteristic (ROC) curve was obtained by plotting the detection rate vs. the false-positive rate using various threshold values.
} 
implementation, demands lower processing power, and requires a relatively small training set, dictated our choice to prefer it to deep learning in the implementation of the baby cry detection module. In all, we thus progressed in accurately capturing a baby cry through a detection algorithm, which will then allow us to more accurately model co-regulation between caregiver peripheral temperature and infant cry.

\subsection{Measurement Module 4: Experience Sampling (Self-Report)}

A classic - and justified - criticism of relying on physiological correlates (like peripheral temperature or electrodermal activity) alone is that they are underspecified to understand psychological states (see classical discussions by Cannon, 1927; James, 1884). Increases in peripheral temperature could for example indicate excitement, affection, or anger (Ekman, Levenson, \& Friesen, 1983). Understanding co-regulatory patterns (with peripheral temperature from a partner or crying of an infant) already aids in better extracting meaning of said physiological correlates. However, just measuring physiological co-regulatory patterns is still insufficient, therefore self-report remains crucial in understanding such physiological dynamics.

We have advocated for a focus on allostatic balance and co-regulation as guiding principles for mobile social physiology and have pointed to the importance of distribution of vigilance processing and temperature regulation as the goals of the organism. We have also briefly alluded to the fact that these processes can become habituated into individual differences like attachment styles (Vergara et al., 2018). These individual difference styles broadly - manifest in secure (when the social environment is reliably supportive), anxious (when the social environment is unpredictably supportive), or avoidant (when the social environment is not supportive) attachment (Fraley et al., 2000). From our perspective, attachment styles can be more generally understood as a form of "predictive coding" of 
allostatic balance, a principle central to understanding self-reported states in relation to coregulation (Beckes et al., 2015).

Predictive coding can be understood as a "multi-level bidirectional cascade, of "topdown" probabilistic generative models with the core predictive coding strategy of efficient encoding and transmission" (Clark, 2013, p. 183). Central to efficient encoding and transmission is - as we have advocated throughout this article - survival and regulation of metabolic resources. These resources can - as a rule of thumb - be understood through an “economy of action" (i.e., more energy needs to come in than exerted; Proffitt, 2006). Bonding to other animals has thus generally evolved - at least in altricial animals - to support infant survival and to cope with the environment more efficiently.

Beyond considerable evidence in altricial animals other than humans, this view is supported by research in human subjects in the fields of co-regulation (Gottman \& Levenson, 1992), social thermoregulation (IJzerman \& Hogerzeil, 2017), and social support (Beckes \& Coan, 2011). Even in humans it is clear that avoidant individuals consume more glucose and have higher fasting glucose levels (Ein-Dor et al., 2015), and that people who are avoidant are less energized by loved ones (Vergara et al., 2018). There is also considerable evidence of the importance of monitoring one's own bodily needs for interpersonal bonding (Troisi, D’Argenio, Peracchio, \& Petti, 2001). Predictive coding for these processes can be more accurately explained through something that has become known as the "Free Energy Principle" (FEP).

The Free Energy Principle (FEP) has its roots in "thermodynamic free energy". Its central underlying idea is that "all (viable) biological organisms resist a tendency to disorder as shown by their homeostatic properties and must therefore minimize the occurrence of events which are atypical ('surprising') in their habitable environment” (Buckley, Kim, McGregor, \& Seth, 2017, p. 2). By minimizing the amount of stress and temperatures 
deviating from homeostatic levels, the organism minimizes the unknowable (thereby minimizing amount of resources needed for such deviations known as "free energy").

As a core principle, we see partner responsiveness, commitment, and other selfreported states or traits in close relationships, as falling under FEPs; insofar as these selfreported states relate to minimizing the occurrence of atypical fluctuations of the environment, specifically pertaining to those which used to be life-threatening and which are central to attachment processes (stress and cold temperatures). The way to incorporate selfreported states is to ask participants at regular intervals about their moods, their perception of their own and their partner's responsiveness and commitment (see Hofmann et al., 2012; Thai \& Page-Gould, 2017). We included a form of experience sampling in our integrated app. We anticipate these aspects of co-regulation to become even more important for more complex social interactions, such as interactions in social groups (like work teams).

Thus, as a general starting principle, we suggest that experience sampling studies in the field of interpersonal relationships start taking into account 1) co-regulatory strategies and 2) predictive coding accounts and free energy principles more generally (Friston, 2010). It is possible that these principles do not hold, but by providing the methods and principles, we have provided falsifiable departure points.

\section{Reflection}

\subsection{Causality}

There is one topic we have avoided until now and that is causality. All of the methods we have described thus far will only allow for indirect causal inferences. Methods in psychology are predicated on the notion that experimental manipulations allow for inferences that a change in one variable leads to a change in another. The methods we have described do not allow for that. However, once co-regulatory dynamics have been charted, it will become 
possible to engage in hypothesis-driven research through confirmatory testing of experimental manipulations. Even the equipment is becoming available for this.

For example, the "EMBR Wave" is an actuator technology, that can manipulate peripheral temperature at the wrist in participants' daily life and could be pre-programmed based on descriptives from our deep learning approach, depending on what is necessary to create a functional co-regulatory pattern in a relationship (EMBR Labs, 2017). The last author's lab has developed scripts in PsychoPy to manipulate peripheral temperature via the EMBR wave via the computer, and connecting the EMBR Wave to the BAB is a goal for the near future. Similarly, the "Doppel" is a wearable device that has been successfully shown to manipulate heartbeat-like tactile stimulation to alter stress levels before public speaking (Azevedo et al., 2017). Thus, not only observing through sensor technology is now possible (through our app and our data-driven methods), manipulating in real-time (and responsive to the partner) through actuator technology is realistic in the near future. The applications for (relationship) therapy are easy to imagine, and within reach.

\section{Conclusion}

Psychological science finds itself at an interesting cross-road. We are faced with problems related to reproducibility. The focus thus far has been at low-powered samples and data-contingent analyses. To better understand interpersonal relationships, we have shifted the focus to an overarching framework (co-regulation of vigilance processing and temperature) and have introduced innovative methods and programmed an app to better understand how co-regulation functions across a variety of interpersonal relationships. It is clear that this is anything but simple and it should be very clear (if only by the broad composition of our author team) that this puzzle can only be solved by expertise from various disciplines. Though psychology may be in crisis, the future is bright, and we anticipate progress to be quick if our research embraces novel technologies. 


\section{References}

Agnew, C. R., Van Lange, P. A., Rusbult, C. E., \& Langston, C. A. (1998). Cognitive interdependence: Commitment and the mental representation of close relationships. Journal of Personality and Social Psychology, 74, 939.

Alois, J. D. (2014). Updated: Backers rebel as Bonaverde Coffee Machine alters design post crowdfunding campaign. [Product Review]. Retrieved from: https://www.crowdfundinsider.com/2014/09/49558-backers-rebel-bonaverde-coffeemachine-alters-design-post-crowdfunding-campaign/.

Andersen, S. M., Moskowitz, G. B., Blair, I. V., \& Nosek, B. A. (2007). Automatic thought. In A. W. Kruglanski, \& E. T. Higgins (Eds.), Social Psychology: Handbook of Basic Principles (pp. 138-175). New York: The Guilford Press.

Awam, K. A., Catana, F., \& Mortola, J. P. (2011). Thermogenic and vocalization responses to cold in the chicken hatchling during normoxia and hypoxia. Behavioral Neuroscience, 125, 74.

Azevedo, R. T., Bennett, N., Bilicki, A., Hooper, J., Markopoulou, F., \& Tsakiris, M. (2017). The calming effect of a new wearable device during the anticipation of public speech. Nature Scientific Reports, 7.

Bargh, J. A. (1994). The four horsemen of automaticity: Intention, awareness, efficiency, and control as separate issues. In R. Wyer \& T. Srull (Eds.), Handbook of Social Cognition (pp. 169 - 183). New York: The Guilford Press.

Barsalou, L. W. (1999). Perceptions of perceptual symbols. Behavioral and Brain Sciences, 22, 637-660.

Barsalou, L. W. (2008). Grounded cognition. Annual Review of Psychology, 59, 617 645. 
Beckes, L., \& Coan, J. A. (2011). Social baseline theory: The role of social proximity in emotion and economy of action. Social and Personality Psychology Compass, 5, 976-988.

Beckes, L., IJzerman, H., \& Tops, M. (2015). Toward a radically embodied neuroscience of attachment and relationships. Frontiers in Human Neuroscience, 9.

Bem, D. J. (2011). Feeling the future: experimental evidence for anomalous retroactive influences on cognition and affect. Journal of Personality and Social Psychology, 100, 407.

Benedek, M., \& Kaernbach, C. (2010a). Decomposition of skin conductance data by means of nonnegative deconvolution. Psychophysiology, 47, 647-658.

Benedek, M., \& Kaernbach, C. (2010b). A continuous measure of phasic electrodermal activity. Journal of Neuroscience Methods, 190, 80-91.

Bishop, C. M. (2006). Pattern Recognition and Machine Learning. New York: Springer.

Bongers, C. W. G., Hopman, M. T. E., \& Eijsvogels, T. M. H. (2017). Validity and reliability of the myTemp ingestible temperature capsule. Journal of Science and Medicine in Sport, 21, $322-326$.

Bowlby, J. (1969). Attachment and Loss. New York: Basic Books.

Brunswik, E. (1956). Perception and the representative design of psychological experiments (2nd ed.). Berkeley, CA, US: University of California Press.

Buckley, C. L., Kim, C. S., McGregor, S., \& Seth, A. K. (2017). The free energy principle for action and perception: A mathematical review. arXiv preprint arXiv:1705.09156. 
Butler, E. A., \& Randall, A. K. (2013). Emotional coregulation in close relationships. Emotion Review, 5, 202-210.

Cannon, W. B. (1927). The James-Lange theory of emotions: A critical examination and an alternative theory. The American Journal of Psychology, 39, 106-124.

Cataletto, M., Angula, M., Hertz, G., \& Whitman, B. (2011). Prader-Willi syndrome: A primer for clinicians. International Journal of Pediatric Endocrinology, 2011, 12.

Cesario, J., \& Jonas, K. J. (2014). Replicability and models of priming: What a resource computation framework can tell us about expectations of replicability. Social Cognition, 32, 124-136.

Chartrand, T. L., \& Bargh, J. A. (1999). The chameleon effect: the perception-behavior link and social interaction. Journal of Personality and Social Psychology, 76, 893.

Cheung, I., Campbell, L., LeBel, E. P., Ackerman, R. A., Aykutoğlu, B., Bahník, Š., . . . Yong, J. C. (2016). Registered Replication Report: Study 1 from Finkel, Rusbult, Kumashiro, \& Hannon (2002). Perspectives on Psychological Science, 11, 750-764.

Clark, A. (2013). Whatever next? Predictive brains, situated agents, and the future of cognitive science. Behavioral and Brain Sciences, 36, 181-204.

Coan, J. A., Schaefer, H. S., \& Davidson, R. J. (2006). Lending a hand: Social regulation of the neural response to threat. Psychological Science, 17, 1032-1039.

Cohen, R., \& Lavner, Y. (2012). Infant cry analysis and detection. In Electrical \& Electronics Engineers in Israel (IEEEI), 2012 IEEE 27th Convention of (pp. 1-5). IEEE.

Comstock, J. (2016). Open source wearable Angel shuts down. Mobi Health News. Retrieved from: http://www.mobihealthnews.com/content/open-source-wearable-angel-shutsdown. 
De Geus, E. J., \& Van Doornen, L. J. (1996). Ambulatory assessment of parasympathetic/sympathetic balance by impedance cardiography. In J. Fahrenberg \& M. Myrtek (Eds), Ambulatory Assessment: Computer-assisted Psychological and Psychophysiological Methods in Monitoring and Field Studies (pp. 141-163). Seattle, WA: Hogrefe and Huber.

Deng, H., Sun, Y., Chang, Y., \& Han, J. (2015). Probabilistic models for classification. In C. C. Aggarwal (Eds.), Data Classification, Algorithms and Applications (pp. 65-86). London: Chapman \& Hall.

Ebensperger, L. A. (2001). A review of the evolutionary causes of rodent group-living. Acta Theriologica, 46, 115-144.

Ebersole, C. R., Atherton, O. E., Belanger, A. L., Skulborstad, H. M., Allen, J. M., Banks, J. B., ... \& Brown, E. R. (2016). Many Labs 3: Evaluating participant pool quality across the academic semester via replication. Journal of Experimental Social Psychology, 67, 68-82.

Ein-Dor, T., Coan, J. A., Reizer, A., Gross, E. B., Dahan, D., Wegener, M. A., ... \& Zohar, A. H. (2015). Sugarcoated isolation: Evidence that social avoidance is linked to higher basal glucose levels and higher consumption of glucose. Frontiers in Psychology, 6.

Ekman, P., Levenson, R. W., Friesen, W. V. (1983). Autonomic nervous system activity distinguishes among emotions. Science, 221, 1208-1210.

EMBR Labs (2017). Your own personal thermostat. Retrieved 2017 from https://embrlabs.com/.

Fiedler, K., Bluemke, M., \& Unkelbach, C. (2009). Exerting control over allegedly automatic associative processes. In J. P. Forgas, R. F. Baumeister, \& D. M. Tice 
(Eds.), Psychology of Self-Regulation: Cognitive, Affective, and Motivational Processes (pp. 249-269). New York: Taylor \& Francis Group.

Finkel, E. J. (2016). Reflections on the commitment-forgiveness registered replication report. Perspective on Psychological Science, 11, 765-767.

Finkel, E. J., Fitzsimons, G. M., \& vanDellen, M. R. (2015). Self-regulation as a transactive process: Reconceptualizing the unit of analysis for goal setting, pursuit, and outcomes. In K. D. Vohs, \& R. F. Baumeister (Eds.), Handbook of Self-Regulation (3 ${ }^{\text {rd }}$ edition). New York: Guilford.

Finkel, E. J., Rusbult, C. E., Kumashiro, M., \& Hannon, P. A. (2002). Dealing with betrayal in close relationships: Does commitment promote forgiveness? Journal of Personality and Social Psychology, 82, 956.

Fiske, A. P. (2002). Using Individualism and Collectivism to Compare Cultures-A Critique of the Validity and Measurement of the Constructs: Comment on Oyserman et al.(2002). Psychological Bulletin, 128, 78-88.

Fraley, R. C., Waller, N. G., \& Brennan, K. A. (2000). An item response theory analysis of self-report measures of adult attachment. Journal of Personality and Social Psychology, 78, 350 .

Francis, G. (2012). Publication bias and the failure of replication in experimental psychology. Psychonomic Bulletin \& Review, 19, 975-991.

Frederiks, K., Croes, M., Chen, W., Bambang Oetomo, S., \& Sterkenburg, P. (2015). Sense a biofeedback system to support the interaction between parents and their child with the Prader-Willi syndrome: A pilot study. Journal of Ambient Intelligence \& Smart Environments, 7, 449-459.

Friston, K. (2010). The free-energy principle: a unified brain theory? Nature Reviews Neuroscience, 11, 127-138. 
Friston, K., \& Kiebel, S. (2009). Predictive coding under the free-energy principle.

Philosophical Transactions of the Royal Society of Londen B: Biological Sciences, $364,1211-1221$.

Gelman, A., \& Loken, E. (2013). The garden of forking paths: Why multiple comparisons can be a problem, even when there is no "fishing expedition" or "p-hacking" and the research hypothesis was posited ahead of time. Unpublished Manuscript, Columbia University. Retrieved from http://www.stat.columbia.edu/ gelman/research/unpublished/p_hacking.pdf.

Giakoumis, D., Tzovaras, D., \& Hassapis, G. (2013). Subject-dependent biosignal features for increased accuracy in psychological stress detection. International Journal of Human-Computer Studies, 71, 425-439.

Goodfellow, I., Bengio, Y., \& Courville A. (2016). Deep Learning. Cambridge, MA: MIT Press.

Gottman, J. M., \& Levenson, R. W. (1992). Marital processes predictive of later dissolution: Behavior, physiology, and health. Journal of Personality and Social Psychology, 63, 221.

Häfner, M., \& IJzerman, H. (2011). The face of love: Spontaneous accommodation as social emotion regulation. Personality and Social Psychology Bulletin, 37, 1551-1563.

Hofmann, W., Baumeister, R. F., Förster, G., \& Vohs, K. D. (2012). Everyday temptations: An experience sampling study of desire, conflict, and self-control. Journal of Personality and Social Psychology, 102, 1318.

IJzerman, H., Coan, J. A., Wagemans, F. M., Missler, M. A., van Beest, I., Lindenberg, S., \& Tops, M. (2015). A theory of social thermoregulation in human primates. Frontiers in Psychology, 6 . 
IJzerman, H., Čolić, M. V., Hennecke, M., Hong, Y., Hu, C. P., Joy-Gaba, J., ... \& Schubert, T. W. (2017). Does distance from the equator predict self-control? Lessons from the Human Penguin Project. Behavioral and Brain Sciences, 40.

IJzerman, H., Gallucci, M., Pouw, W. T., Weißgerber, S. C., Van Doesum, N. J., \& Williams, K. D. (2012). Cold-blooded loneliness: social exclusion leads to lower skin temperatures. Acta Psychologica, 140, 283-288.

IJzerman, H., Heine, E. C., Nagel, S. K., \& Pronk, T. M. (2017). Modernizing relationship therapy through Social Thermoregulation Theory: Evidence, hypotheses, and explorations. Frontiers in Psychology, 8.

IJzerman, H., \& Hogerzeil, L. (2017). People as penguins: Thermoregulation as part of the human essence. In M. van Zomeren \& J. Dovidio (Eds.). The Handbook of Human Essence. Oxford University Press.

IJzerman, H., Janssen, J. A., \& Coan, J. A. (2015). Maintaining warm, trusting relationships with brands: Increased temperature perceptions after thinking of communal brands. PloS one, 10, e0125194.

IJzerman, H., Ligtenberg, W., \& Verbeke, W. (2017). The Social Thermoregulation App. Retrieved from: https://github.com/wligtenberg/closeness-android.

IJzerman, H., Lindenberg, S., Dalğar, I., Weissgerber, S. C., Vergara, R. C., Cairo, A., .. \& \& Hall, C. (2018). The Human Penguin Project: Social Integration protects against cold climates. Collabra: Psychology. Preprint available at PsyArxiv: https://osf.io/preprints/psyarxiv/6b7ne.

IJzerman, H., Pollet, T., Ebersole, C., \& Kun, D. (2017). What predicts Stroop performance? A conditional random forest approach. Preprint available at PsyArxiv: https://osf.io/8t437/. 
IJzerman, H., Pollet, T. V., Kun, D., \& Ebersole, C. R. (2017). What predicts Stroop performance? [Script] Retrieved from osf.io/x53pu.

Ioannidis, J. P. (2005). Why most published research findings are false. PLoS medicine, 2, e124.

James, W. (1884). What is an emotion? Mind, 9, 188-205.

Joel, S., Eastwick, P. W., \& Finkel, E. J. (2018). Open sharing of data on close relationships and other sensitive social psychological topics: Challenges, tools, and future directions. Advances in Methods and Practices in Psychological Science, 1, 86-94.

Kirkpatrick, L. A. (1998). God as a substitute attachment figure: A longitudinal study of adult attachment style and religious change in college students. Personality and Social Psychology Bulletin, 24, 961-973.

Kirschbaum, C., Pirke, K. M., \& Hellhammer, D. H. (1993). The 'Trier Social Stress Test' A tool for investigating psychobiological stress responses in a laboratory setting. Neuropsychobiology, 28, 76-81.

Klein, O., Hardwicke, T. E., Aust, F., Breuer, J., Danielsson, H., Mohr, A. H., ... \& Frank, M. C. (2018). A practical guide for transparency in psychological science. Collabra: Psychology, 4 .

Klein, R. A., Ratliff, K. A., Vianello, M., Adams, R. B., Bahník, Š., Bernstein, M. J., ... \& Cemalcilar, Z. (2014). Investigating variation in replicability. Social Psychology, 45, 142-152.

Klein, R. A., Vianello, M., Hasselman, F., Adams, B. G., Adams, R. B., Alper, S., \& Friedman, M. (2018). Many Labs 2: Investigating variation in replicability across sample and setting. Manuscript in preparation.

Krebs, J. R., Davies, N. B., \& Parr, J. (1993). An Introduction to Behavioural Ecology. Hoboken, NJ: Blackwell Scientific Publications. 
Lakens, D. (2014). Grounding social embodiment. Social Cognition, 32, 168-183.

Lavner, Y., Cohen, R., Ruinskiy, D., \& IJzerman, H. (2016). Baby cry detection in domestic environment using deep learning. ICSEE 2016.

LeBel, E. P., \& Campbell, L. (2013). Heightened sensitivity to temperature cues in individuals with high anxious attachment: Real or elusive phenomenon? Psychological Science, 24, 2128-2130.

Likowski, K. U., Mühlberger, A., Seibt, B., Pauli, P., \& Weyers, P. (2008). Modulation of facial mimicry by attitudes. Journal of Experimental Social Psychology, 44, 10651072.

Mallinckrodt, B., Gantt, D. L., \& Coble, H. M. (1995). Attachment patterns in the psychotherapy relationship: Development of the Client Attachment to Therapist Scale. Journal of Counseling Psychology, 42, 307-307.

Mandler, J. M. (1992). How to build a baby: II. Conceptual primitives. Psychological Review, 99, 587.

McCandless, S. E. (2011). Health supervision for children with Prader-Willi syndrome. Pediatrics, 127, 195-204.

McEwen, B. S. (1998). Stress, adaptation, and disease: Allostasis and allostatic load. Annals of the New York Academy of Sciences, 840, 33-44.

Mizukami, K., Kobayashi, N., Ishii, T., \& Iwata, H. (1990). First selective attachment begins in early infancy: A study using telethermography. Infant Behavior and Development, $13,257-271$.

Ogorevc, J., Geršak, G., Novak, D., \& Drnovšek, J. (2013). Metrological evaluation of skin conductance measurements. Measurement, 46, 2993-3001.

Open Science Collaboration. (2015). Estimating the reproducibility of psychological science. Science, 349, aac4716. 
Picard, R. W., Fedor, S., \& Ayzenberg, Y. (2016). Multiple arousal theory and daily-life electrodermal activity asymmetry. Emotion Review, 8, 62-75.

Quatieri, T. F. (2002). Discrete-time speech signal processing: principles and practice. Upper Saddle River, NJ: Prentice Hall.

Reis, H. T., \& Gable, S. L. (2015). Responsiveness. Current Opinion in Psychology, 1, $67-$ 71.

Reus, L., Zwarts, M., van Vlimmeren, L. A., Willemsen, M. A., Otten, B. J., \& Nijhuis-van der Sanden, M. W. G. (2011). Motor problems in Prader-Willi syndrome: A systematic review on body composition and neuromuscular functioning. Neuroscience \& Biobehavioral Reviews, 35, 956-969.

Rusbult, C. E., Olsen, N., Davis, J. L., \& Hannon, P. A. (2004). Commitment and relationship maintenance mechanisms. Close relationships: Key readings, 287 - 303.

Schuengel, C., Sterkenburg, P. S., Jeczynski, P., Janssen, C. G. C., \& Jongbloed, G. (2009). Supporting affect regulation in children with multiple disabilities during psychotherapy: A multiple case design study of therapeutic attachment. Journal of Consulting and Clinical Psychology, 77, 291.

Simmons, J. P., Nelson, L. D., \& Simonsohn, U. (2011). False-positive psychology: Undisclosed flexibility in data collection and analysis allows presenting anything as significant. Psychological Science, 22, 1359-1366.

Smith, E. R., Murphy, J., \& Coats, S. (1999). Attachment to groups: Theory and management. Journal of Personality and Social Psychology, 77, 94.

Smith, E. R., \& Semin, G. R. (2004). Socially situated cognition: Cognition in its social context. Advances in Experimental Social Psychology, 36, 53-117.

Spellman, B. A. (2015). A short (personal) future history of revolution 2.0. Perspective on Psychological Science, 10, 886. 
Srivastava, S. (2016). The Hardest Science. [Blog]. Retrieved from: hardsci.wordpress.com.

Sterkenburg, P., Frederiks, K., Barakova, E., Chen, W., Peters, P. \& Feijs, L. (2017). A bioresponse system for caregivers of adults with severe or profound intellectual disabilities. Journal of Mental Health Research in Intellectual Disabilities, 10, 121.

Sterling, P. (2003). Principles of allostasis: Optimal design, predictive regulation, pathophysiology, and rational therapeutics. In J. Schulkin (Ed.), Allostasis, homeostasis, and the costs of adaptation (pp. 1-35). Cambridge, MA: MIT Press.

Storm, H. (2012). Pain assessment in neonates. Neonatal Monitoring Technologies: Design for Integrated Solutions, IGI Global, 278-302.

Tarus. (2016). Review: Angel Sensor Fitness Tracker [Product Review]. Retrieved from: https://www.adventuresinoss.com/2016/02/20/review-angel-sensor-fitness-tracker/.

Thai, S., \& Page-Gould, E. (2017, June 15). ExperienceSampler: An open-source scaffold for building smartphone apps for experience sampling. Psychological Methods. Advance online publication. http://dx.doi.org/10.1037/met0000151.

Troisi, A., D’Argenio, A., Peracchio, F., \& Petti, P. (2001). Insecure attachment and alexithymia in young men with mood symptoms. The Journal of Nervous and Mental Disease, 189, 311-316.

Van Emden, R. (2015). [Application software]. Retrieved from: https://github.com/robinvanemden/Closeness.

Van Lange, P. A., Rinderu, M. I., \& Bushman, B. J. (2017). Aggression and violence around the world: A model of CLimate, Aggression, and Self-control in Humans (CLASH). Behavioral and Brain Sciences, 40, 1 - 63. 
Van Marken Lichtenbelt, W. D., Daanen, H. A., Wouters, L., Fronczek, R., Raymann, R. J., Severens, N. M., \& Van Someren, E. J. (2006). Evaluation of wireless determination of skin temperature using iButtons. Physiology \& Behavior, 88, 489-497.

Van 't Veer, A. E., Galluci, M., Stel, M., \& Beest, I. (2015). Unconscious deception detection measured by finger skin temperature and indirect veracity judgment - results of a registered report. Frontiers in Psychology, 6.

Várallyay, G. (2007). The melody of crying. International Journal of Pediatric Otorhinolaryngology, 71, 1699-1708.

Várallyay, G. (2009). Analysis of the Infant Cry with Objective Methods. Unpublished doctoral dissertation, Budapest University of Technology and Economics, Hungary.

Vergara, R. C., Hernández, C., Jaume-Guazzini, F., Lindenberg, S., Klein, R. A., \& IJzerman, H. (2018). Presentation and validation of the Social Thermoregulation and Risk Avoidance Questionnaire (STRAQ-1). Unpublished dataset.

Vinkers, C. H., Penning, R., Hellhammer, J., Verster, J. C., Klaessens, J. H., Olivier, B., \& Kalkman, C. J. (2013). The effect of stress on core and peripheral body temperature in humans. Stress, 16, 520-530.

Vuorenkoski, V., Wasz-Höckert, O., Koivisto, E., \& Lind, J. (1969). The effect of cry stimulus on the temperature of the lactating breast of primipara. A thermographic study. Cellular and Molecular Life Sciences, 25, 1286-1287.

Yarkoni, T., \& Westfall, J. (2017). Choosing prediction over explanation in psychology: Lessons from machine learning. Perspectives in Psychological Science, 1100 - 1122. 
Figures

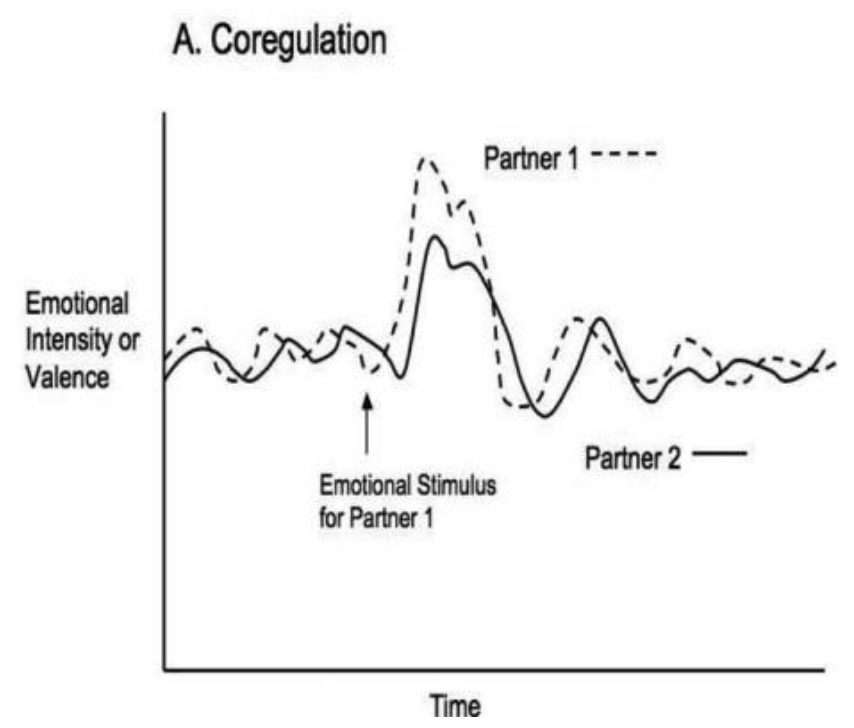

\section{B. Morphogenic Process}

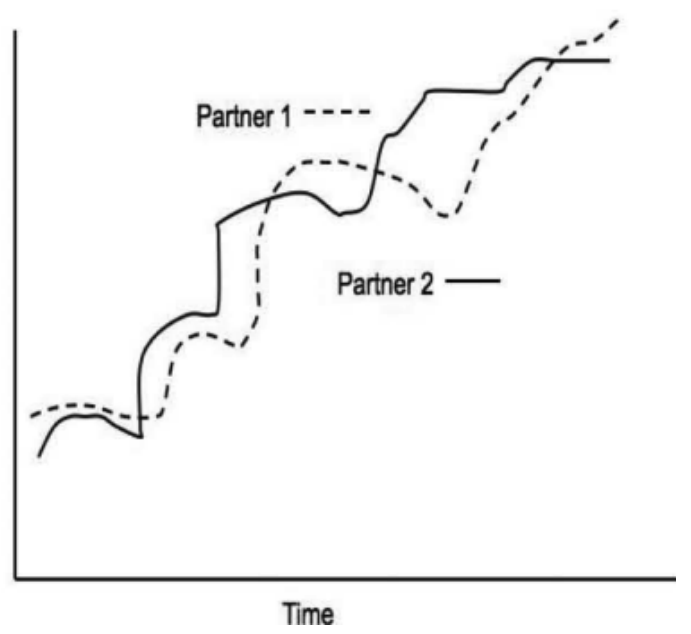

Figures $1 A$ and $B$. Panel $1 \mathrm{~A}$ shows a hypothetical example of co-regulation with the goal of homeostatic regulation. Panel 1B shows an example of mimicking (such as in the case of negative reciprocity in a close relationship), which is also referred to as a morphogenic process. Mimicking negative emotional responses is possible, for example, in case of low relationship quality (taken from Butler \& Randall, 2013). 

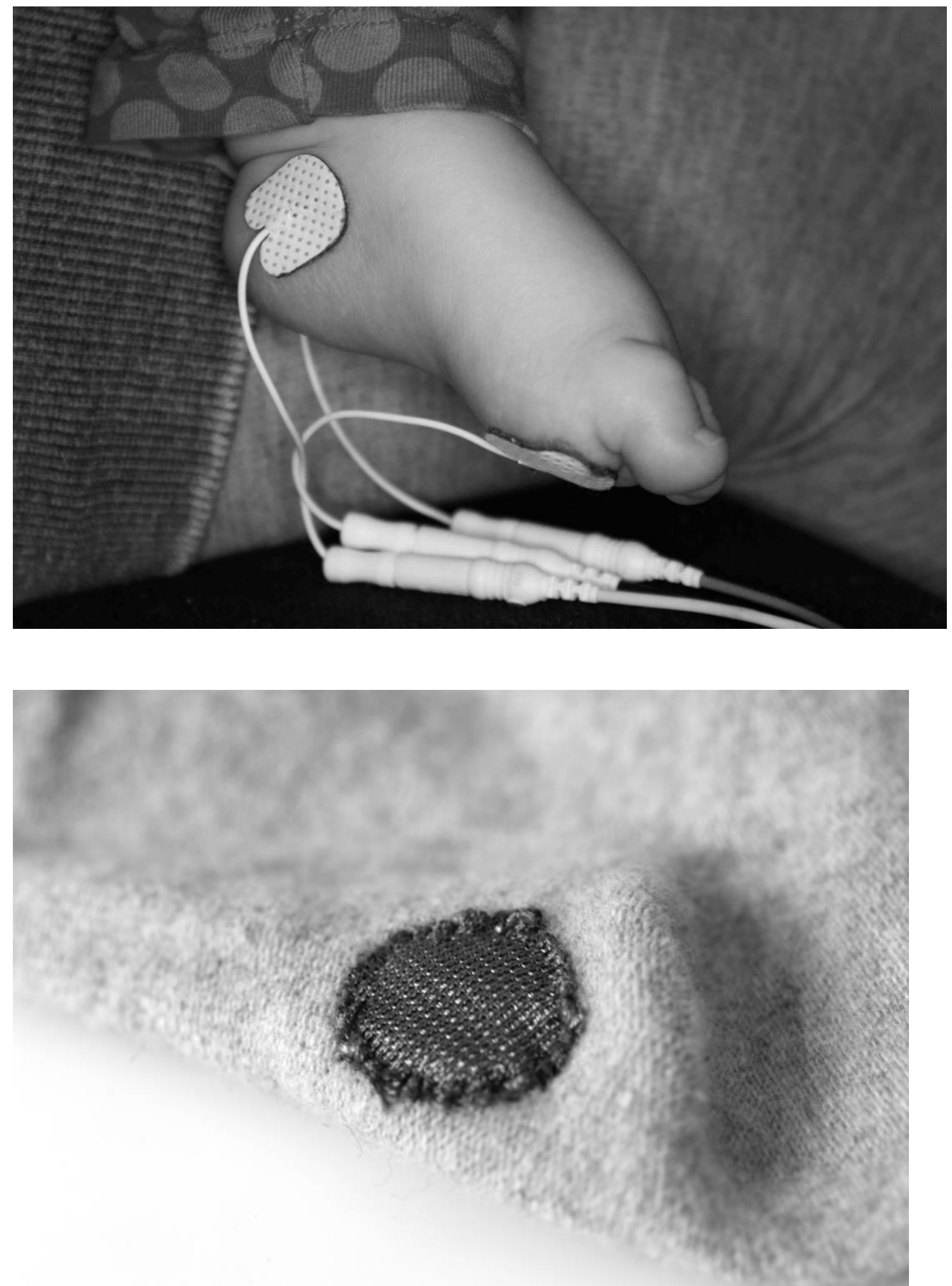

Figure 2. Panel 2A: Sticky electrodes attached to the child's foot for EDA measurements while using the Med-Storm Pain Monitor. Panel 2B: Fabric electrodes (replacing the gel electrodes) integrated in the sole of a sock for comfortable EDA measurements with the Shimmer ${ }^{\mathrm{TM}} 2 \mathrm{R}$ BioPhysical module. 


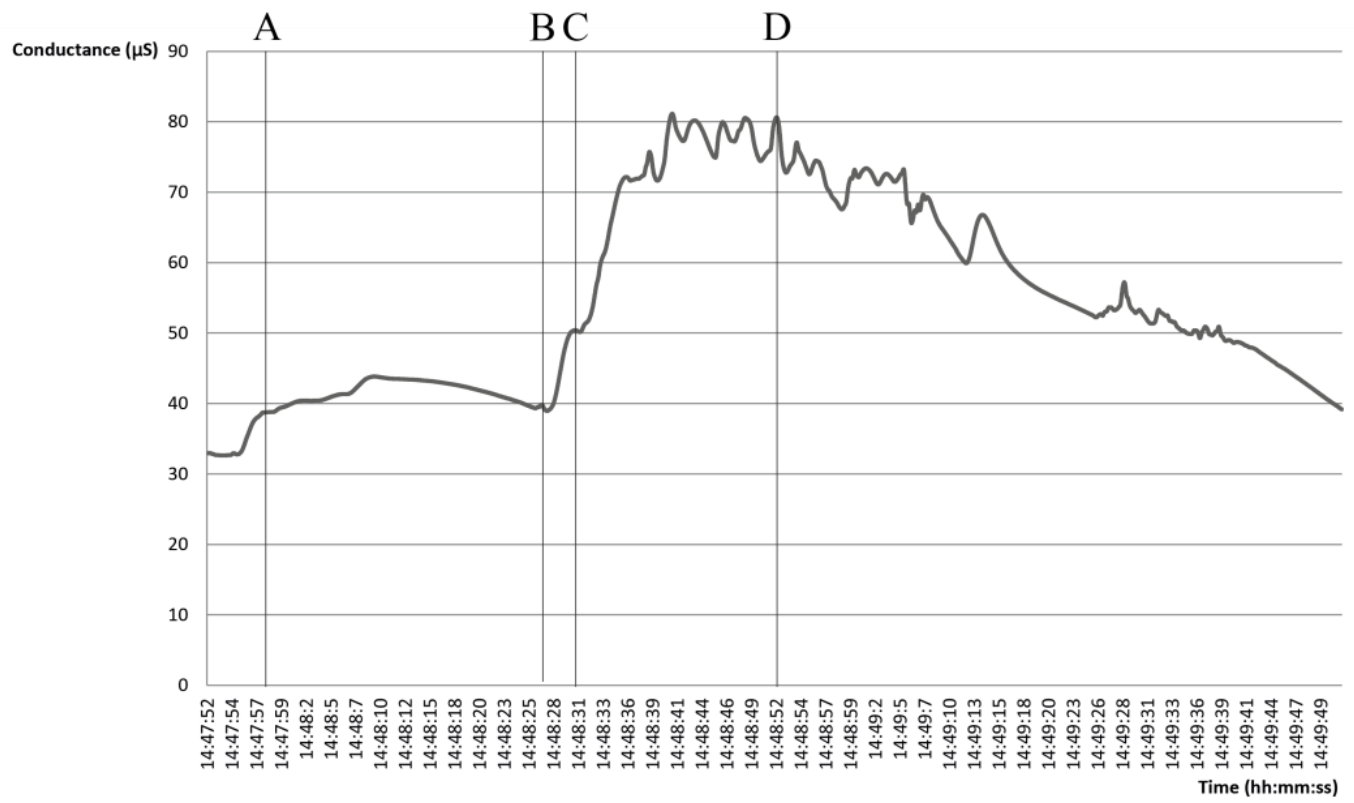
A: The mother places the child on her lap
B: The mother applies the nose spray the one of the child's nostril
C: The child starts crying
D: The mother applies the nose spray the other nostril

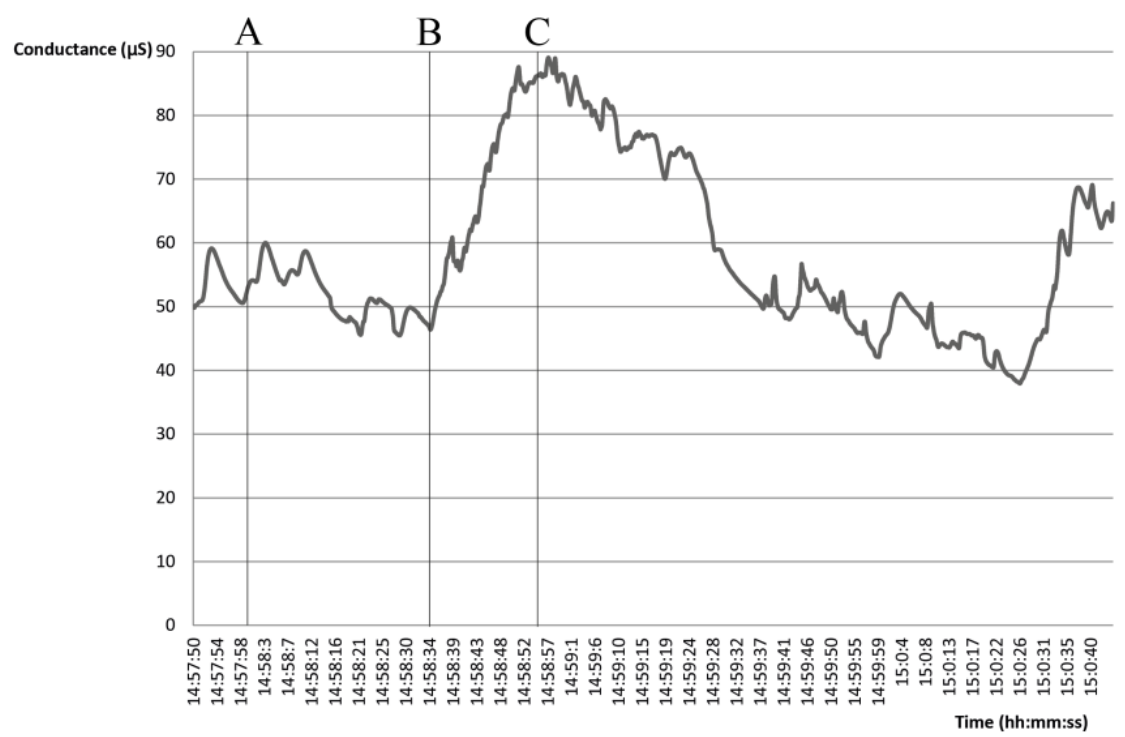

A: The mother lifts the child above her head

B: The mother 'throws' the child a little distance in the air.

$\mathrm{C}$ : The mother 'throws' the child a little distance in the air.

Figure 3. Panel 3A shows an example of general arousal of a child during an unpleasant daily care moment of applying a nose spray. Panel 3B is an example of the child's general arousal levels during a fun play moment together with the mother (from: Frederiks et al., 2015). 


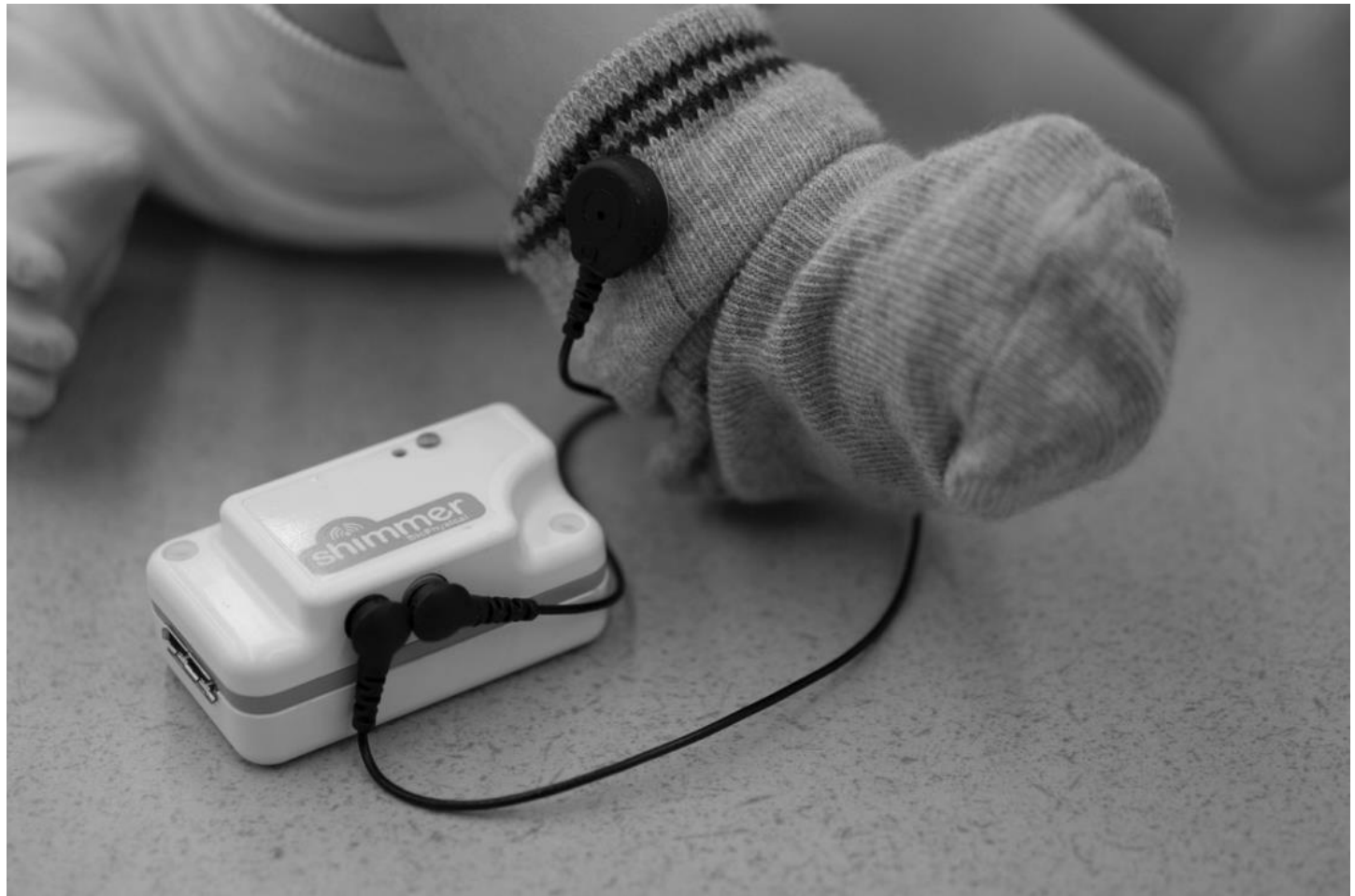

Figure 4. The Shimmer ${ }^{\mathrm{TM}}$ 2R BioPhysical sensor module connected to the snap fasteners of skin conductance (SC) sensor sock.

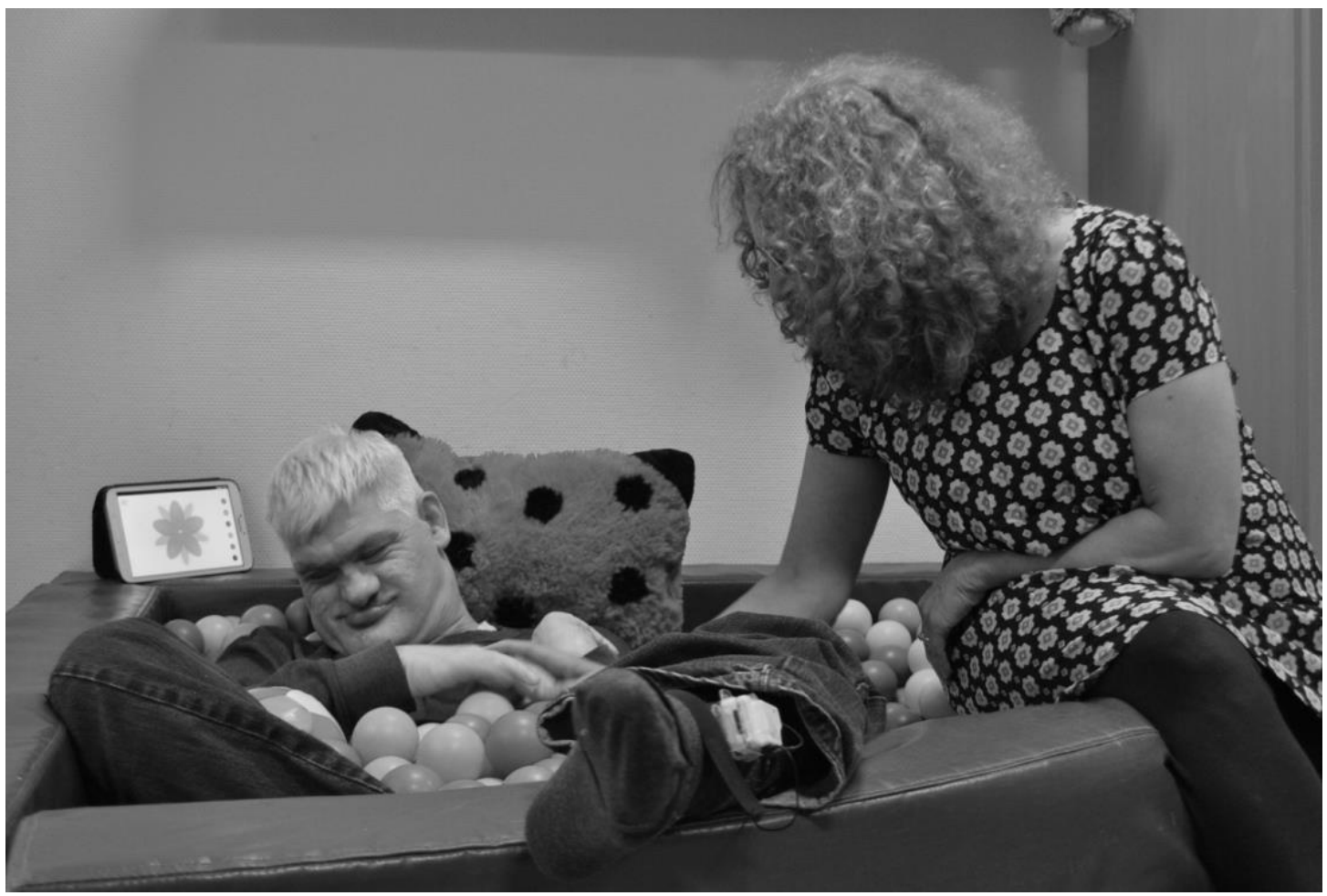

Figure 5. The Sense system used in pilot study with an adult with a visual and severe intellectual disability and his caregiver. 


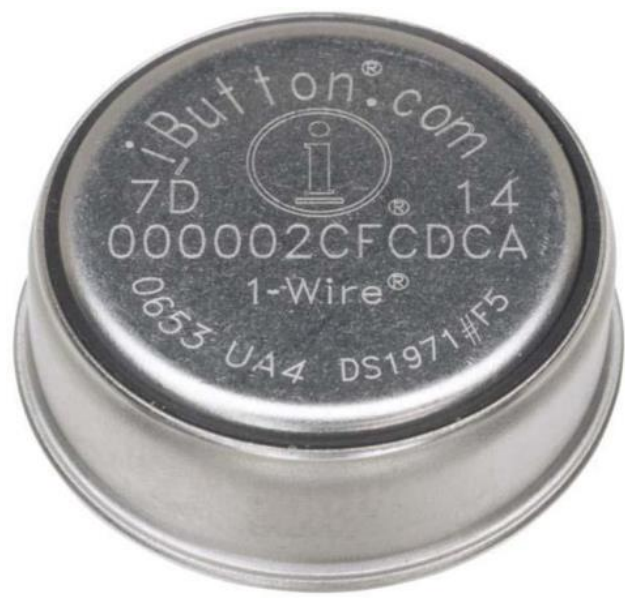

Figure 6. Maxim's Thermochron iButton DS1291H.

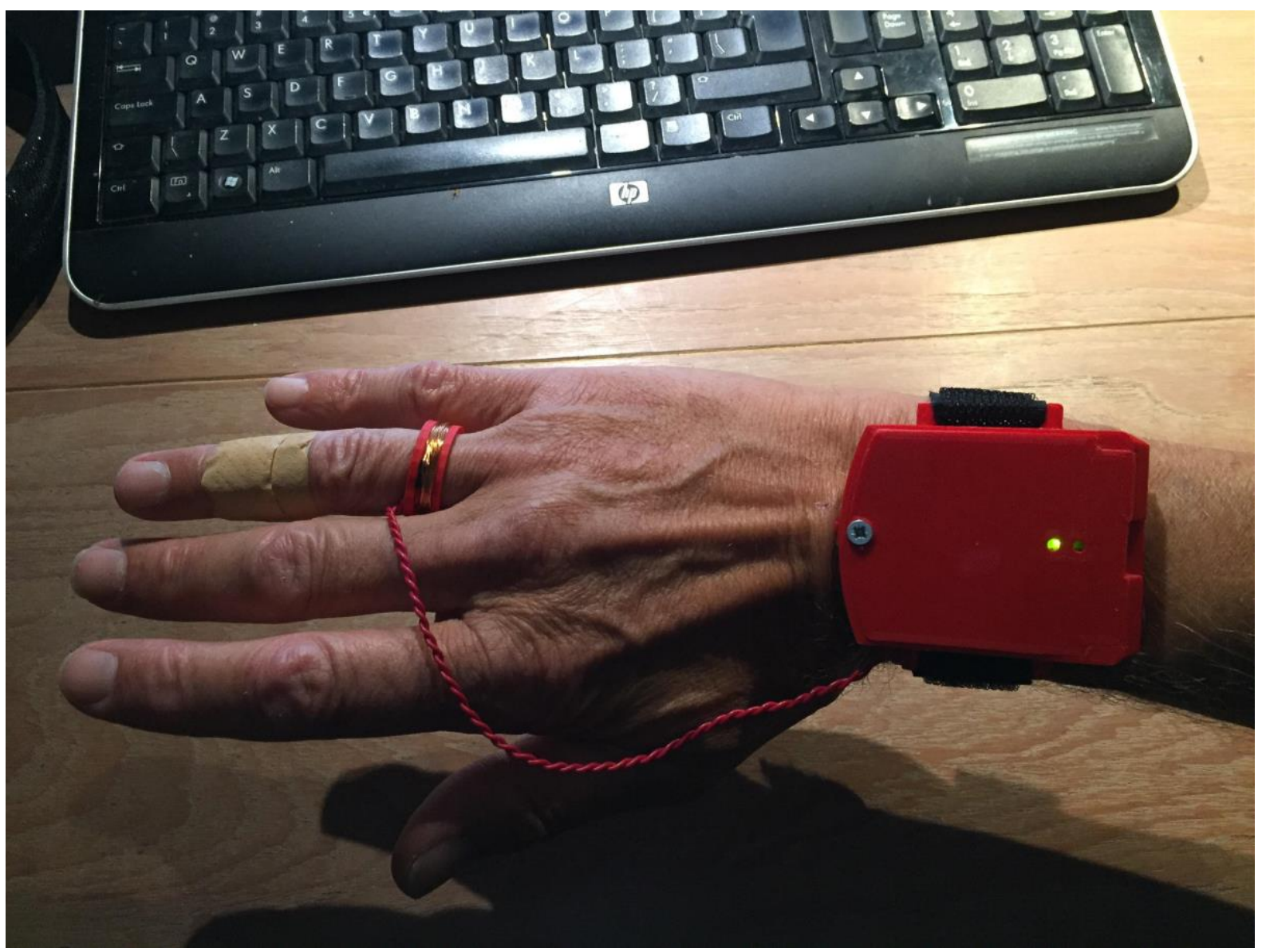

Figure 7. MyTemp Sensor. The sensor is attached to the finger via tape. The wristband functions to charge the sensor. 

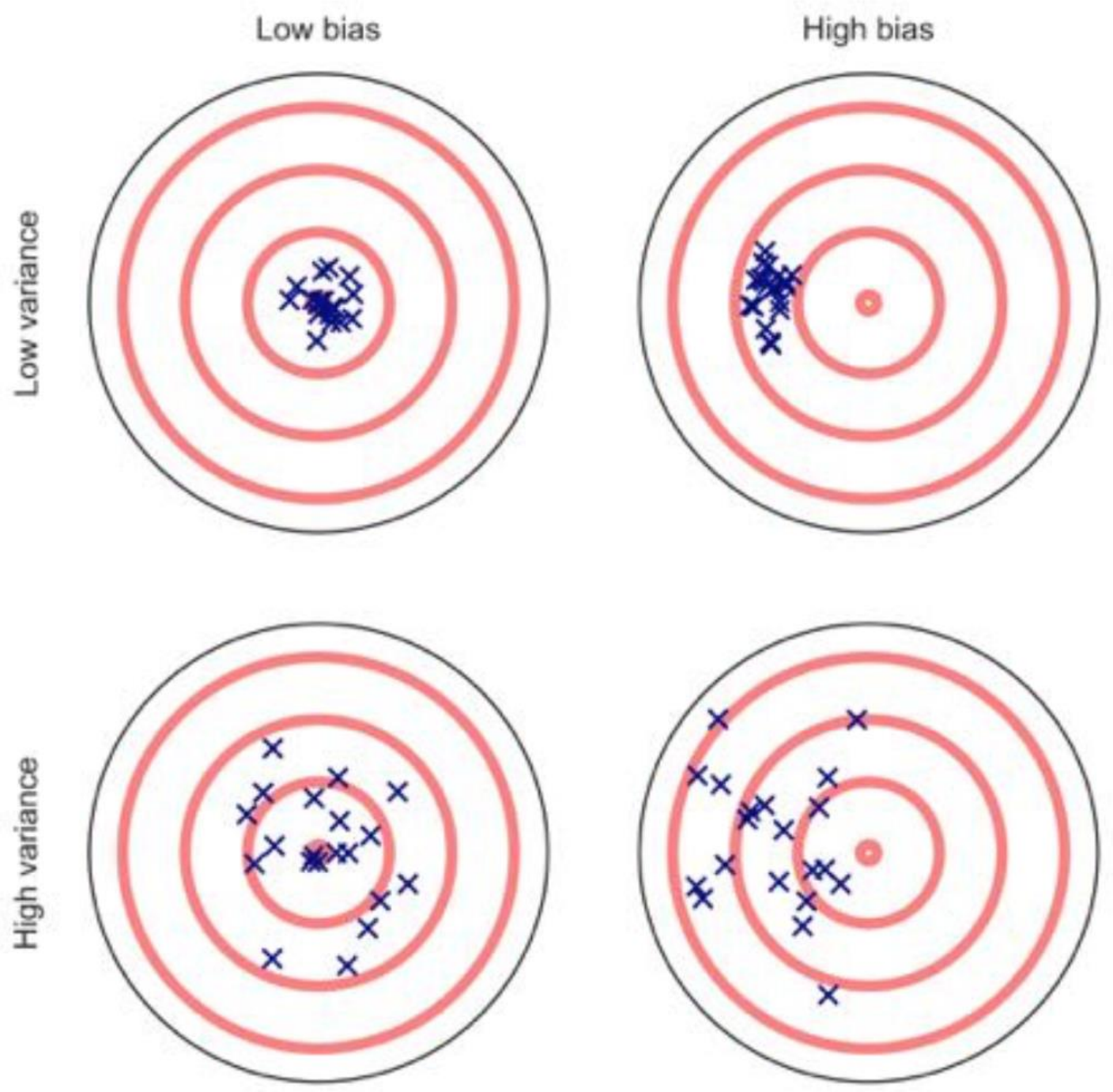

Figure 8. Because of convergent methods of study, we suspect that the relationship literature, overall, has produced a literature with low bias but high variance (lower left corner), with individual researchers producing high bias and low and high variance (right side). Bias is defined as a systematic tendency to deviate from true scores, whereas variance is defined as imprecision in estimates (From Yarkoni \& Westfall, 2017). 

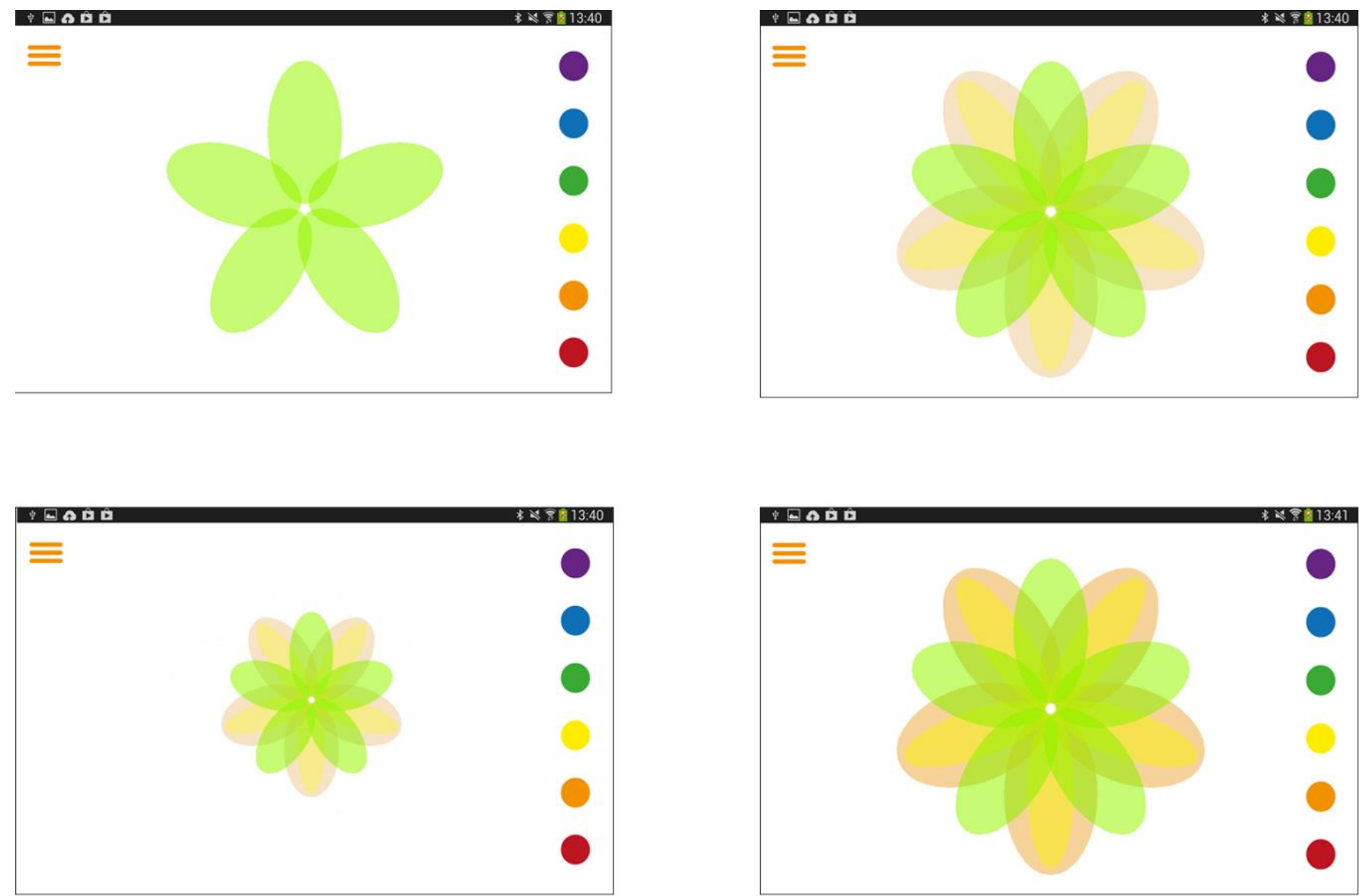

Figure 9. Flower app - Visualization of the skin conductance signal in the form of a flower. Top left: high arousal, low reaction to stimuli; Top right: high arousal, medium reaction to stimuli; Bottom left: low arousal, high reaction to stimuli; Bottom right: high arousal, high reaction to stimuli. 


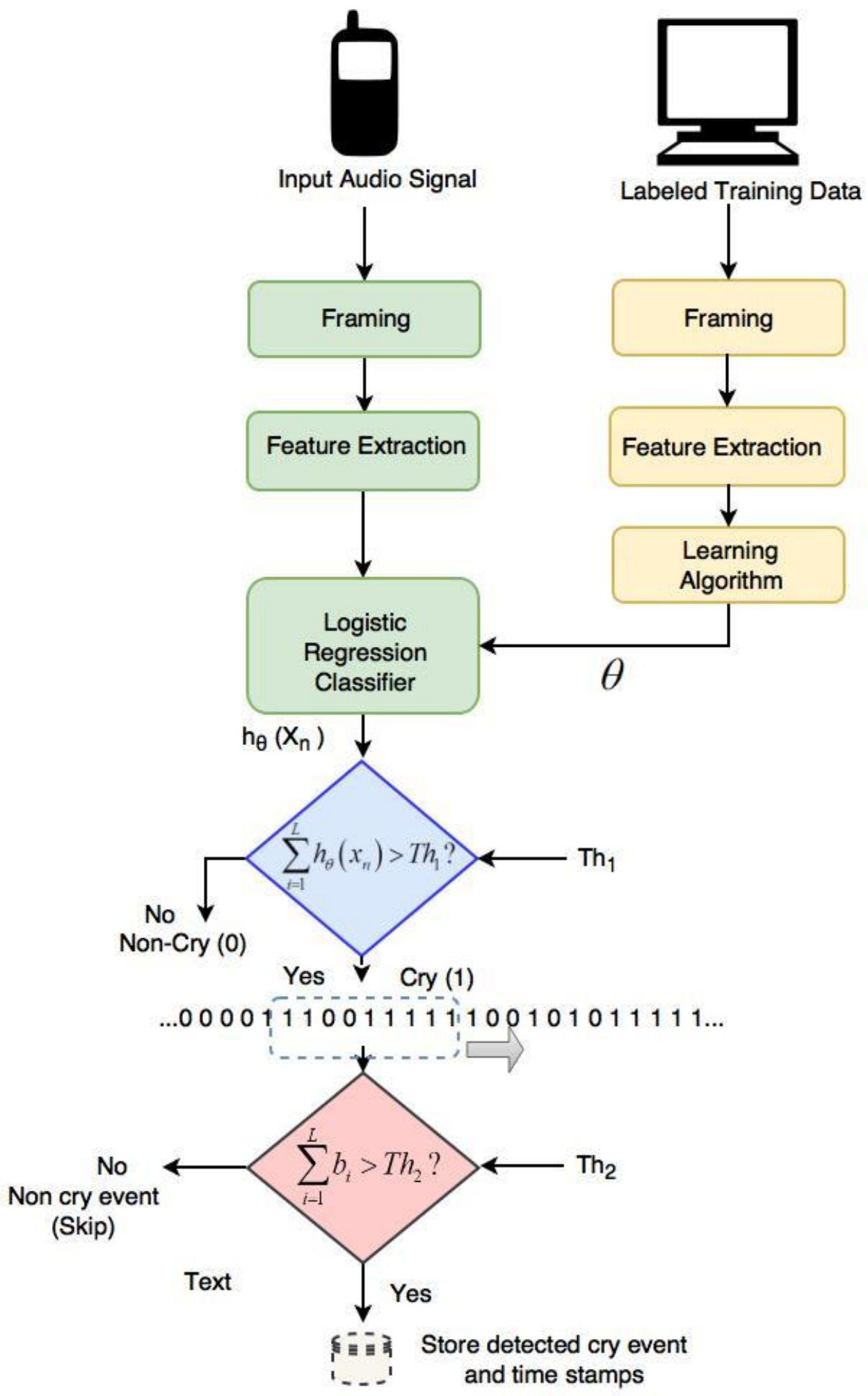

Figure 10. A block diagram of the supervised machine learning detection algorithm. 

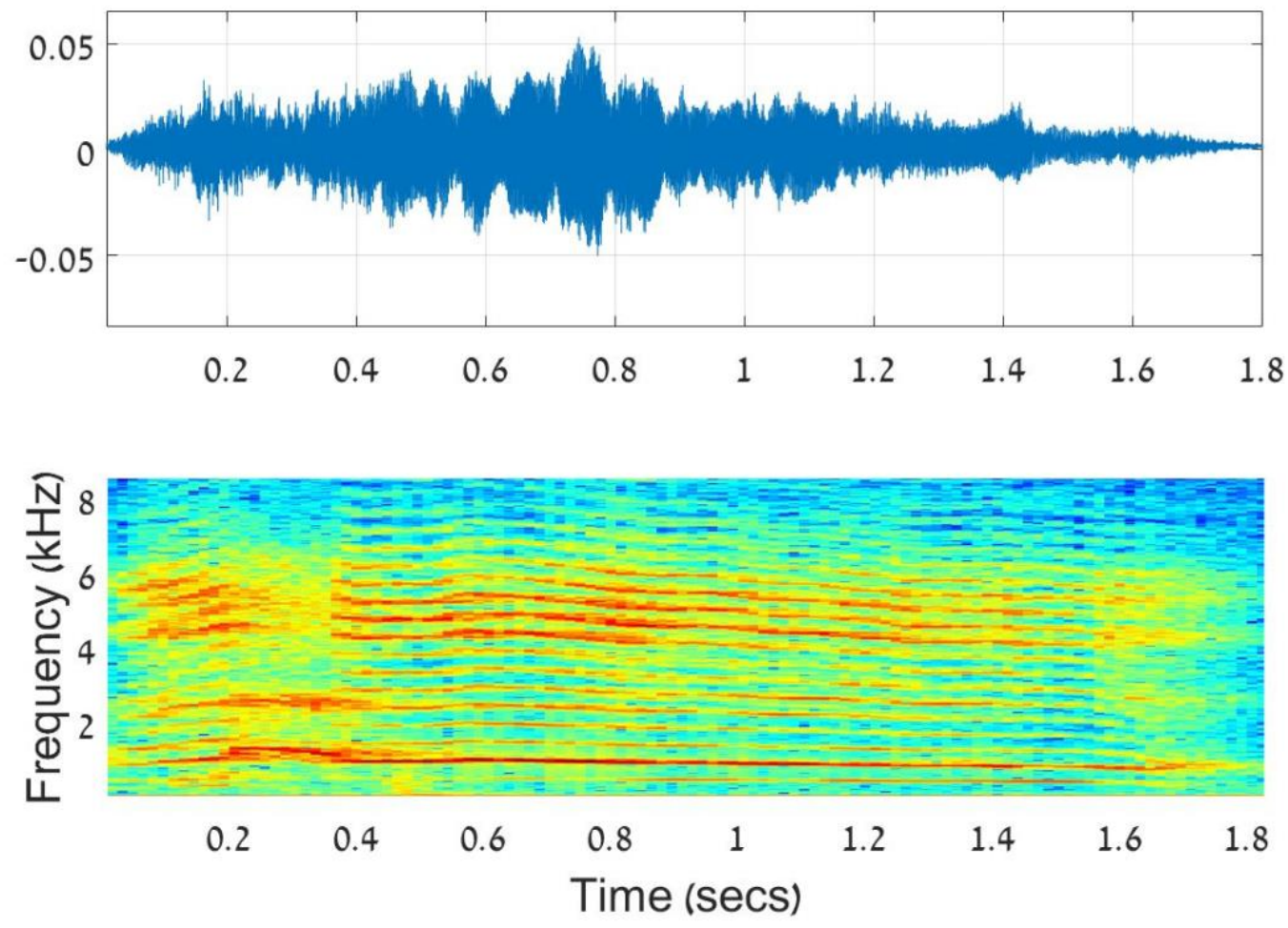

Figure 11. Representation of the infant cry: Top panel: The time waveform of an audio signal that contains a single cry burst. Bottom panel: The corresponding (narrow-band) spectrogram, characterized by a harmonic structure, where each horizontal line represents a partial harmonic. This partial harmonic is nearly an integer multiple of the fundamental frequency. 


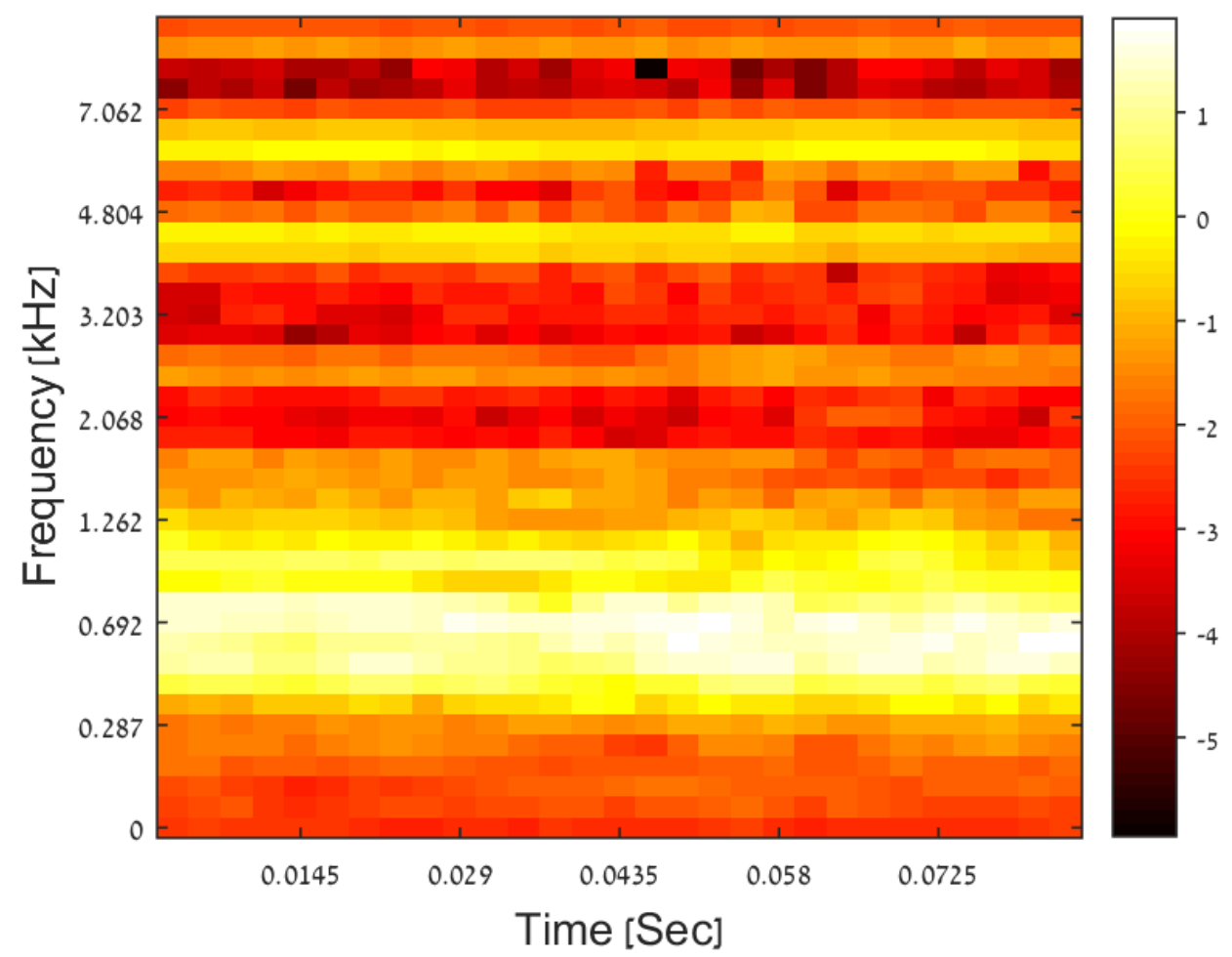

Figure 12. Log Mel-filter bank representation of a cry signal used as input to a convolutional neural network $(\mathrm{CNN})$ for classification purposes.

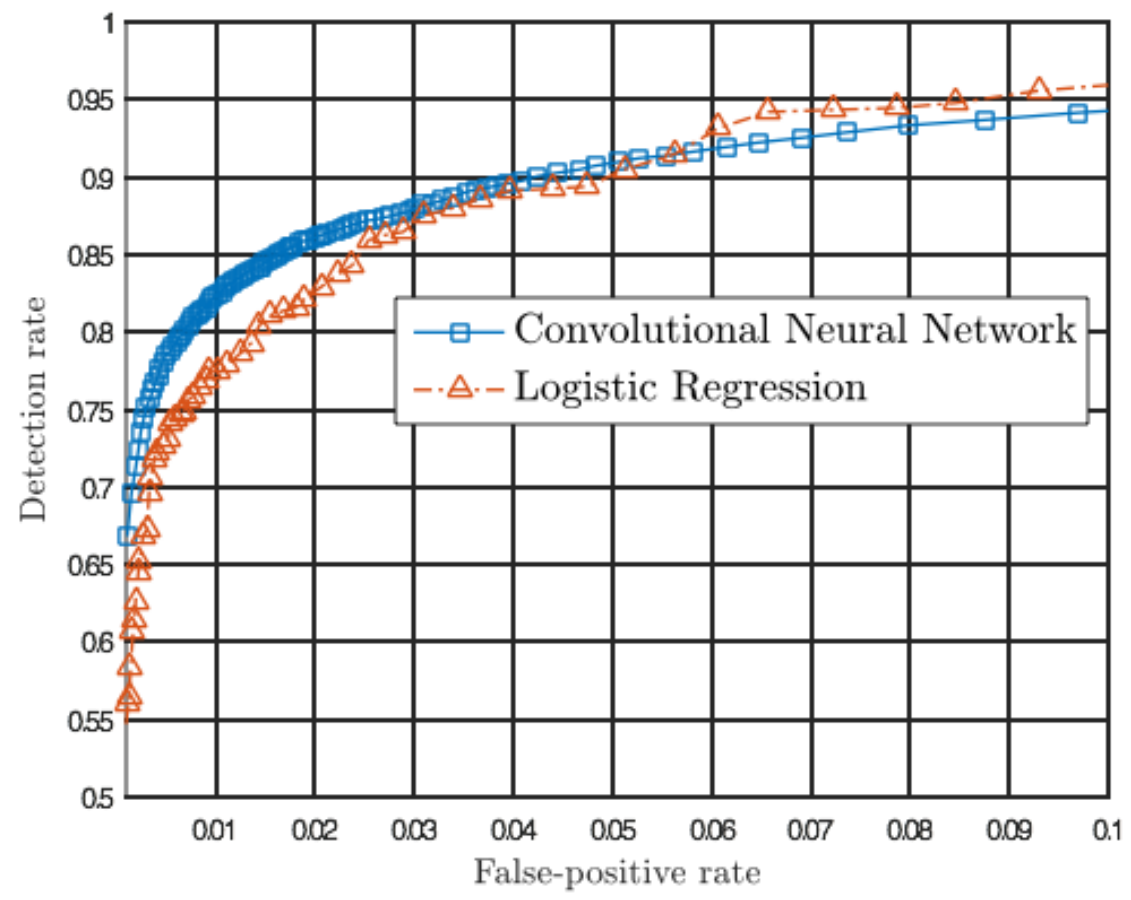

Figure 13. Receiver Operator Characteristic Curves for the logistic regression (red curve, triangles) and the CNN classifiers (blue curve, rectangles). 